\title{
Parkes HI observations of galaxies behind the southern Milky Way
}

\section{The Crux and Great Attractor regions $\left(I \approx 289^{\circ} \text { to } 338^{\circ}\right)^{\star, \star \star}$}

\author{
A. C. Schröder ${ }^{1,2}$, R. C. Kraan-Korteweg ${ }^{3}$, and P. A. Henning ${ }^{4}$ \\ 1 Hartebeesthoek Radio Astronomy Observatory, PO Box 443, Krugersdorp 1740, South Africa \\ e-mail: anja@hartrao.ac.za \\ 2 Dept. of Physics and Astronomy, University of Leicester, University Road, Leicester LE1 7RH, UK \\ 3 Department of Astronomy, University of Cape Town, Private Bag X3, Rondebosch 7701, South Africa \\ ${ }^{4}$ Institute for Astrophysics, University of New Mexico, MSC07 4220, 800 Yale Blvd., NE, Albuquerque, NM, 87131, USA
}

Received 28 May 2009 / Accepted 15 July 2009

\begin{abstract}
As part of our programme to map the large-scale distribution of galaxies behind the southern Milky Way, we observed 314 opticallyselected, partially-obscured galaxies in the Zone of Avoidance (ZOA) in the Crux and Great Attractor (GA) regions. An additional 29 galaxies were observed in the Vela ZOA survey region (because of the small numbers they are not discussed any further). The observations were conducted with the Parkes $64 \mathrm{~m}(210 \mathrm{ft})$ radio telescope, in a single-pixel pointed mode, reaching an rms noise level of typically $2-6 \mathrm{mJy}$ over the velocity search range of $400<v<10500 \mathrm{~km} \mathrm{~s}^{-1}$. A total of 162 galaxies were detected (plus 14 galaxies in the Vela region). The detection rate is slightly higher than for the Hydra/Antlia region (52\% versus $45 \%)$ observed in the same way. This can be explained by the prominence of the GA overdensity in the survey regions, which leads to a relatively higher fraction of nearby galaxies. It is also evident from the quite narrow velocity distribution (largely confined to $3000-6000 \mathrm{~km} \mathrm{~s}^{-1}$ ) and deviates significantly from the expectation of a uniform galaxy distribution for the given sensitivity and velocity range. No systematic differences were found between detections and non-detections, in terms of latitude, foreground extinction, or environment, except for the very central part of the rich Norma cluster, where hardly any galaxies were detected. A detailed investigation of the H I content of the galaxies reveals strong H I deficiency at the core of the Norma cluster (within about a 0.4 Abell radius), similar to what has been found in the Coma cluster. The redshifts obtained by this observing technique result in a substantial reduction of the so-called redshift ZOA. This is obvious when analysing the large-scale structure of the new H I data in combination with data from other (optical) ZOA redshift surveys. The lower latitude detections provide further evidence of the extension of the Norma Wall, across the ZOA, in particular its bending towards the Cen-Crux clusters above the Galactic plane at slightly higher redshift, rather than a straight continuation towards the Centaurus clusters.
\end{abstract}

Key words. catalogs - surveys - ISM: dust, extinction - galaxies: fundamental parameters - radio lines: galaxies cosmology: large-scale structure of Universe

\section{Introduction}

Revealing the three-dimensional distribution of galaxies over the entire sky including the regions behind the dust and stars of our Milky Way is important for understanding the motion of the Local Group with respect to the microwave background as well as the peculiar flow fields in the nearby Universe (e.g., review by Kraan-Korteweg \& Lahav 2000; Kraan-Korteweg 2005; and contributions in "Mapping the Hidden Universe", 2000, ASP CS 218, eds. Kraan-Korteweg et al. 2000; "Nearby Large-Scale Structures and the Zone of Avoidance", 2005, ASP CS 329, eds. Fairall \& Woudt 2005). Except for blind H I surveys where both the angular coordinates and redshifts of galaxies are simultaneously detected, this is a two-step process: first the galaxies have to be identified (in the optical, near or far-infrared), then

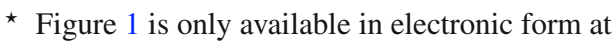
http: //www . aanda.org

$\star \star$ Table 1 is also available in electronic form at the CDS via anonymous ftp to cdsarc.u-strasbg.fr $(130.79 .128 .5)$ or via http://cdsweb.u-strasbg.fr/cgi-bin/qcat?]/A+A/505/1049
}

redshifts have to be determined in follow-up studies. This has been done in the optical, either as single-channel or multi-fibre spectroscopy depending on surface brightness of the galaxies and the galaxy density on the sky or in the radio using the $21 \mathrm{~cm}$ spectral line of neutral hydrogen. The latter is most effective for the most obscured and/or low surface brightness spirals and irregular galaxies.

Based on the deep optical galaxy catalogues in the southern Zone of Avoidance (ZOA; Kraan-Korteweg 2000; Woudt \& Kraan-Korteweg 2001), we have obtained pointed H I observations of a sample of obscured spiral galaxies with the $64 \mathrm{~m}$ Parkes radio telescope in Australia. Previous results of the Hydra/Antlia region $\left(266^{\circ} \lesssim \ell \lesssim 296^{\circ}\right)$ were presented in Kraan-Korteweg et al. (2002, hereafter Paper I). The second part, presented here, covers the observations of spiral galaxies in the Crux and Great Attractor regions (hereafter GA; $289^{\circ} \lesssim$ $\ell \lesssim 338^{\circ},-10^{\circ} \lesssim b \lesssim+10^{\circ}$; Woudt \& Kraan-Korteweg 2001). The optical search detected galaxies above a diameter limit of $D \gtrsim 0.2$ on IIIaJ film copies of the ESO/SRC sky survey. For a detailed description of the optical search, see Paper I and 
the optical catalogue papers (Kraan-Korteweg 2000; Woudt \& Kraan-Korteweg 2001). In summary, our target list consisted of spiral galaxies without redshift information at the time of observation, which have extinction-corrected diameters larger than $D^{0}>60^{\prime \prime}$ (based on the Schlegel at al. 1998 extinction maps, and the Cameron 1990 correction laws). This corresponds to the completeness limit of the deep optical ZOA galaxy catalogues to foreground extinction levels of $A_{B} \leq 3$. 0 (Kraan-Korteweg 2000). It will therefore complement existing optical wholesky catalogues such as Nilson (1973) for the northern sky and Lauberts (1982) for the southern sky. The ESO/SRC IIIaJ filmcopies that were used for the searches have such fine-grained emulsion and sensitivity that the spiral morphology could always be discerned with our $50 \times$ magnifying viewer - though not always the spiral sub-type. At the highest extinction levels, we also targeted smaller galaxies since optical spectroscopy is unlikely to succeed in yielding redshifts for these heavily extincted galaxies. We also sampled deeper in some of the overdensities like, e.g., the Norma cluster.

Our sample has a sensitivity of 2-6 mJy. This study is therefore complementary to the systematic blind H I survey of the southern ZOA conducted with the Parkes multibeam receiver (Staveley-Smith et al. 2000; for preliminary results see Kraan-Korteweg et al. 2005; Henning et al. 2005), which covers the optically opaque part of the southern ZOA $\left(212^{\circ} \leq \ell \leq 36^{\circ}\right.$, $|b| \leq 5^{\circ}$ ) for the velocity range -1200 to $12700 \mathrm{~km} \mathrm{~s}^{-1}$, with a sensitivity similar to the observations presented here. A subsample of this work consists of the shallow H I ZOA survey (hereafter HIZSS; Henning et al. 2000), based on $8 \%$ of the integration time of the full survey with a sensitivity of $15 \mathrm{mJy} \mathrm{beam}^{-1}$ after Hanning smoothing). In another subsample which is based on $16 \%$ of the integration time of the full survey, Juraszek et al. (2000, hereafter JS00) focused on the area of the GA in the ZOA $\left(308^{\circ} \lesssim \ell \lesssim 332^{\circ}\right)$.

In the following section, a short description of the observations is given (see Paper I for further details). Section 3 presents the HI data and line profiles of the detected galaxies. In Sect. 4, the non-detected galaxies are listed with their respective velocity search range. An analysis of the properties of the detected galaxies, the velocity distribution as well as the detection rate (Sect. 5) is followed by a detailed discussion of H I-deficiency in the Norma cluster (Sect. 6). We then present a description of the three-dimensional galaxy distribution in and around the investigated area (Sect. 7). A summary is given in Sect. 8. Throughout the paper we assume a Hubble constant of $H_{0}=70 \mathrm{~km} \mathrm{~s}^{-1} \mathrm{Mpc}^{-1}$.

In Appendix A, we discuss cross-identifications of optical galaxies for which the detected H I signal might not necessarily be associated with the optical counterpart, or where more than one galaxy was detected in one pointing. In Appendix B, we present detections in the Vela region $\left(245^{\circ} \lesssim \ell \lesssim 275^{\circ}\right)$ which have also been observed during the course of these observing runs.

\section{Observations}

The Parkes $64 \mathrm{~m}$ radio telescope ${ }^{1}$ was used over four observing periods of about 10-14 days each (June 1993, April 1994, July 1995 and September 1996). Here, we report on the observations that cover the Crux and GA ZOA regions.

\footnotetext{
1 The Parkes telescope is part of the Australia Telescope which is funded by the Commonwealth of Australia for operation as a National Facility managed by CSIRO.
}

A detailed description of the observational set-up is given in Paper I. A summary of the main characteristics of the observations is given below.

At $21 \mathrm{~cm}$, the telescope has a half-power beam-width $(H P B W)$ of $15^{\prime}$. The system temperature was typically $39 \mathrm{~K}$ at the time of these observations. Typical integration times were a total of 30 min each on the source $(\mathrm{ON})$ and on a reference position (OFF). Strong sources had shorter integration times (10 or $20 \mathrm{~min}$ ) while weaker possible detections were reobserved until the reality of the signal was clearly determined. Based on calibrating observations the internal consistency of the flux scale is about $\pm 15 \%$.

In 1993, we used the 1024-channel auto correlator with a bandwidth of $32 \mathrm{MHz}$, covering, in most cases, the radial velocity range $300-5500 \mathrm{~km} \mathrm{~s}^{-1}$, with some additional observations centred at $7500 \mathrm{~km} \mathrm{~s}^{-1}$; the channel spacing was $6.6 \mathrm{~km} \mathrm{~s}^{-1}$ and the velocity resolution after Hanning smoothing was $13.2 \mathrm{~km} \mathrm{~s}^{-1}$. From 1994 on, we covered the range $300-10500 \mathrm{~km} \mathrm{~s}^{-1}$ with a channel spacing of $13.2 \mathrm{~km} \mathrm{~s}^{-1}$ and a velocity resolution after Hanning smoothing of $27.0 \mathrm{~km} \mathrm{~s}^{-1}$.

\section{Detections}

In the following, we present the parameters of the 162 detected galaxies, from the sample of 314 target galaxies. The data were reduced using the Spectral Line Analysis Program (Staveley-Smith 1985). The two orthogonal polarisations were averaged during reduction, and a low-order polynomial baseline subtracted from each spectrum. The reduced H I spectra are shown in Fig. 1 which is available online at A\&A. The optical properties as well as the H I parameters are given in Table 1. The columns in the table are described below. A colon after an entry indicates an uncertain value.

Column 1: identification as given in the optical Crux/GA ZOA galaxy catalogue (Woudt \& Kraan-Korteweg 2001). A few galaxies from Yamada et al. (1993), which are all IRAS-selected galaxies, had been added to the observing programme. Their names start with "Y" and have the ESO-galaxy number or the IRAS number attached to it. A question mark after the name denotes an uncertain identification of the H I signal, and a plus indicates that more than one signal was found in the pointing or in the associated OFF-observation.

Column 2: second name as found by $\mathrm{NED}^{2}$ in the order of NGC, IC, ESO, and other catalogue names. Most of the second identifications originate from the ESO/Uppsala Survey of the ESO(B) Atlas (Lauberts 1982). Other names come from the following catalogues: NGC stands for the New General Catalogue (Dreyer 1888), IC stands for the Index Catalogue (Dreyer 1908), FGCE stands for the Flat Galaxy Catalog (Karachentsev et al. 1993), AM for Arp \& Madore (1987), CSRG for Catalog of Southern Ringed Galaxies (Buta 1995), HIZSS for the H I Parkes ZOA Shallow Survey (Henning et al. 2000), and HIZOA for Juraszek et al. (2000).

Column 3: identification in the infrared (IR) and nearinfrared (NIR): "I" indicates an entry in the IRAS Point Source Catalog according to the precepts explained in Woudt \& Kraan-Korteweg (2001); "M" or "D" indicates an entry in the 2MASS Extended Objects Catalogue (2MASS, 2003) and the DENIS catalogue by Vauglin et al. (2002), respectively.

Columns 4 and 5: Right Ascension RA and Declination $\operatorname{Dec}(\mathrm{J} 2000.0)$.

Columns 6 and 7: Galactic longitude and latitude $\ell$ and $b$.

\footnotetext{
2 the NASA/IPAC Extragalactic Database.
} 
Column 8: morphological type. The morphological types are coded similarly to the precepts of the RC2 (de Vaucouleurs et al. 1976) with the addition of the subtypes E, M and L, which stand for early spiral (S0/a-Sab), middle spiral ( $\mathrm{Sb}-\mathrm{Sd})$ and late spiral or irregular $(\mathrm{Sdm}-\mathrm{Im})$, respectively. (Note that the uncertainty of the bar presence is indicated by "Y" as in Woudt \& Kraan-Korteweg (2001), rather than the standard " $X$ " as in the $\mathrm{RC} 2$. We have kept their denotation.)

Column 9: major axis diameter $D$ and minor axis diameter $d$ in arcseconds.

Column 10: apparent magnitude $B_{J}$. These magnitudes are eye-estimates from the ESO/SERC IIIaJ film copies. They compare well with the Lauberts \& Valentijn (1989) $B_{25}$ magnitudes and have a $1 \sigma$-dispersion of less than 0.5 .

Column 11: the Galactic reddening at the position of the galaxy, as given by the DIRBE/IRAS extinction maps (Schlegel et al. 1998). See the catalogue paper (Woudt \& Kraan-Korteweg 2001) for a more detailed discussion.

Column 12: heliocentric H I radial velocity in $\mathrm{km} \mathrm{s}^{-1}$ taken at the midpoint of the HI profile at the $20 \%$ level. The velocity is given in the optical convention $V=c \cdot\left(\lambda-\lambda_{o}\right) / \lambda_{o}$. The uncertainty on the velocities, $\sigma_{V}$, can be determined following Schneider et al. (1986) using $1.5\left(\Delta V_{20}-\Delta V_{50}\right)(S / N)^{-1}$, where $S / N$ is the ratio of the peak signal to rms noise level. The median error over all detections is $3.6 \mathrm{~km} \mathrm{~s}^{-1}$.

Column 13: velocity width in $\mathrm{km} \mathrm{s}^{-1}$ of the H I profile measured at the $50 \%$ level of the peak intensity. The expected error is $2.0 \sigma_{V}$ (Schneider et al. 1986), and the median error derived from the table is $7.3 \mathrm{~km} \mathrm{~s}^{-1}$.

Column 14: velocity width in $\mathrm{km} \mathrm{s}^{-1}$ of the H I profile measured at the $20 \%$ level of the peak intensity. The expected error is $3.1 \sigma_{V}$ (Schneider et al. 1986), and the median error derived from the table is $11.3 \mathrm{~km} \mathrm{~s}^{-1}$.

Column 15: integrated H I flux density, in $\mathrm{Jy} \mathrm{km} \mathrm{s}^{-1}$, uncorrected for finite beam size. The uncertainty on the flux densities, $\sigma_{I}$, can be determined following Schneider et al. (1990) using $2\left(1.2 \Delta V_{20} / R\right)^{0.5} R \sigma$, where $R$ is the velocity resolution of the data (see Sect. 2) and $\sigma$ is the rms noise level. The median error over all defined flux densities is $0.65 \mathrm{Jy}$.

Column 16: rms noise level in mJy measured over the region used to fit a baseline, typically of a width of $1600 \mathrm{~km} \mathrm{~s}^{-1}$ centred on, but not including, the detection.

Column 17: most spectra have been Hanning-smoothed, except when the line width was smaller than $100 \mathrm{~km} \mathrm{~s}^{-1}$ or for other reasons (see footnotes to the table), which is indicated with an "n" for "no Hanning-smoothing".

Column 18: a star indicates a footnote for this entry.

Column 19: angular distance of the detected galaxy from the centre of the beam in arcminutes. Sometimes the telescope was not pointed towards the galaxy listed as, e.g., in a close pair or when there was a second detection in the beam. As the sensitivity of the beam decreases with distance to the beam centre, the fluxes will have higher uncertainties (see Col. 20).

Column 20: corrected flux densities for off-centre detections. The sensitivity decreases as a function of the distance from the beam centre. The observed flux density of galaxies detected away from the central pointing will be underestimated. We have provided a rough correction for such galaxies by assuming that the beam sensitivity can be approximated by a circular Gaussian. This correction becomes uncertain for distances above the beam radius, i.e., 7 '.5.

Column 21: excised RFI (radio frequency interference) on or near the detected H I profile.
The galaxy density in the Crux and particular in the GA region is quite high. In $16 \%$ of the pointings, more than one galaxy was found within the $1 \sigma$ beam radius. In most cases the proper identification of the detected galaxy was straightforward (by comparing size, morphological type, optical velocity if available, and distance from the beam centre). In questionable cases we have made use of the HIPASS public data release ${ }^{3}$ (as a blind survey it is independent of our pointed observations) to specify the origin of a signal. In a few cases the identification of the detection remains ambiguous or the counterpart could not be found at all. Due to the high extinction in these regions, low surface brightness (late-type) spiral galaxies are often too obscured to be visible. In other cases the detection is a combination of the signals from two or even more galaxies and the individual $\mathrm{H}$ I parameters are uncertain or could not be derived. The problematic cases are discussed in more detail in Appendix A.

We have compared our detected velocities with independent velocity determinations in the literature. Table 2 gives the galaxy IDs for which we have found velocity determinations, their observed velocity (from Col. 12 in Table 1), the velocity from the literature and its error, the origin of the measurement (optical or HI), and the reference (as explained in Table 3).

Since we did not repeat observations at Parkes for galaxies with already existing H I data, most of the given independent velocities originate from optical spectroscopy. A number of strong H I sources were subsequently detected with HIZSS, JS00 and/or with HIPASS.

The H I velocities measured by HIPASS with the Multibeam (MB) receiver compared to our single beam observations are in very good agreement. The $1 \sigma$-dispersion of 31 galaxies in common is $11 \mathrm{~km} \mathrm{~s}^{-1}$ (including only velocities with HIPASS error measurements $<10 \mathrm{~km} \mathrm{~s}^{-1}$ ). A comparison with HIZSS gives a dispersion of $6.5 \mathrm{~km} \mathrm{~s}^{-1}$ for 7 galaxies. The agreement with optical velocities is also satisfactory, giving a dispersion of $100 \mathrm{~km} \mathrm{~s}^{-1}$ for 50 measurements with errors $<100 \mathrm{~km} \mathrm{~s}^{-1}$ (excluding one wrong measurement for WKK 5416).

The line width measurements also agree well with HIPASS albeit with a larger scatter of $28 \mathrm{~km} \mathrm{~s}^{-1}$ for both $50 \%$ and $20 \%$ line widths (using 29 measurements, excluding all uncertain measurements).

While the shallower HIPASS survey detected all galaxies with peak flux density $>120 \mathrm{mJy}$, the majority of our detections have peak flux densities $<70 \mathrm{mJy}$, see Fig. 2. Most of the HIPASS detections below $70 \mathrm{mJy}$ have larger uncertainties, while our survey becomes incomplete only at $<20 \mathrm{mJy}$. This compares well with the distribution of rms noise in our survey: the median rms level lies at $3.8 \mathrm{mJy}$. The majority of detections have an rms noise level between 2 and $6 \mathrm{mJy}$. For comparison, the rms noise for HIPASS typically is $13 \mathrm{mJy}$, though expected to be slightly higher in the Galactic plane. This indicates that we are sensitive enough to detect $M^{*}$ galaxies in the GA region contrary to the blind HIPASS.

Comparing integrated flux densities with HIPASS gives a $1 \sigma$-dispersion of $25 \%$ of the flux for 23 measurements (uncertain measurements excluded). Reducing the acceptable HIPASS error on velocities from $9 \mathrm{~km} \mathrm{~s}^{-1}$ to $8 \mathrm{~km} \mathrm{~s}^{-1}$ (cf. the comparison of $\mathrm{H}$ I velocities above) the $1 \sigma$-dispersion is $15 \%$ of the flux (for 19 measurements), which is more comparable with what we find for the calibrators (cf. Sect. 2). Table 1 lists 14 galaxies offset from the beam centre. A comparison of the corrected fluxes

\footnotetext{
3 Data provided by the ATNF under the auspices of the Multibeam Survey Working Group, see http://www.atnf.csiro.au/ research/multibeam/release/
} 


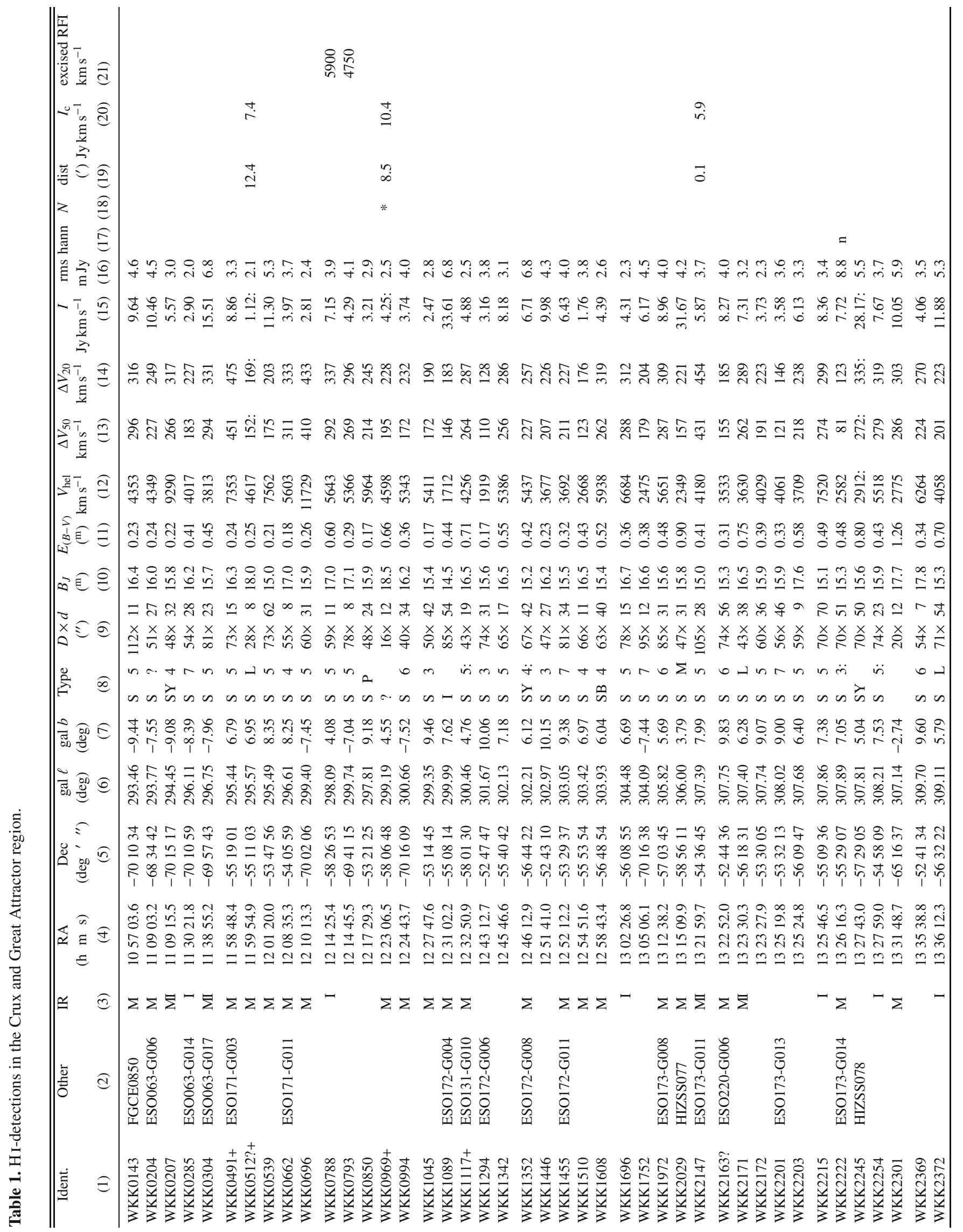




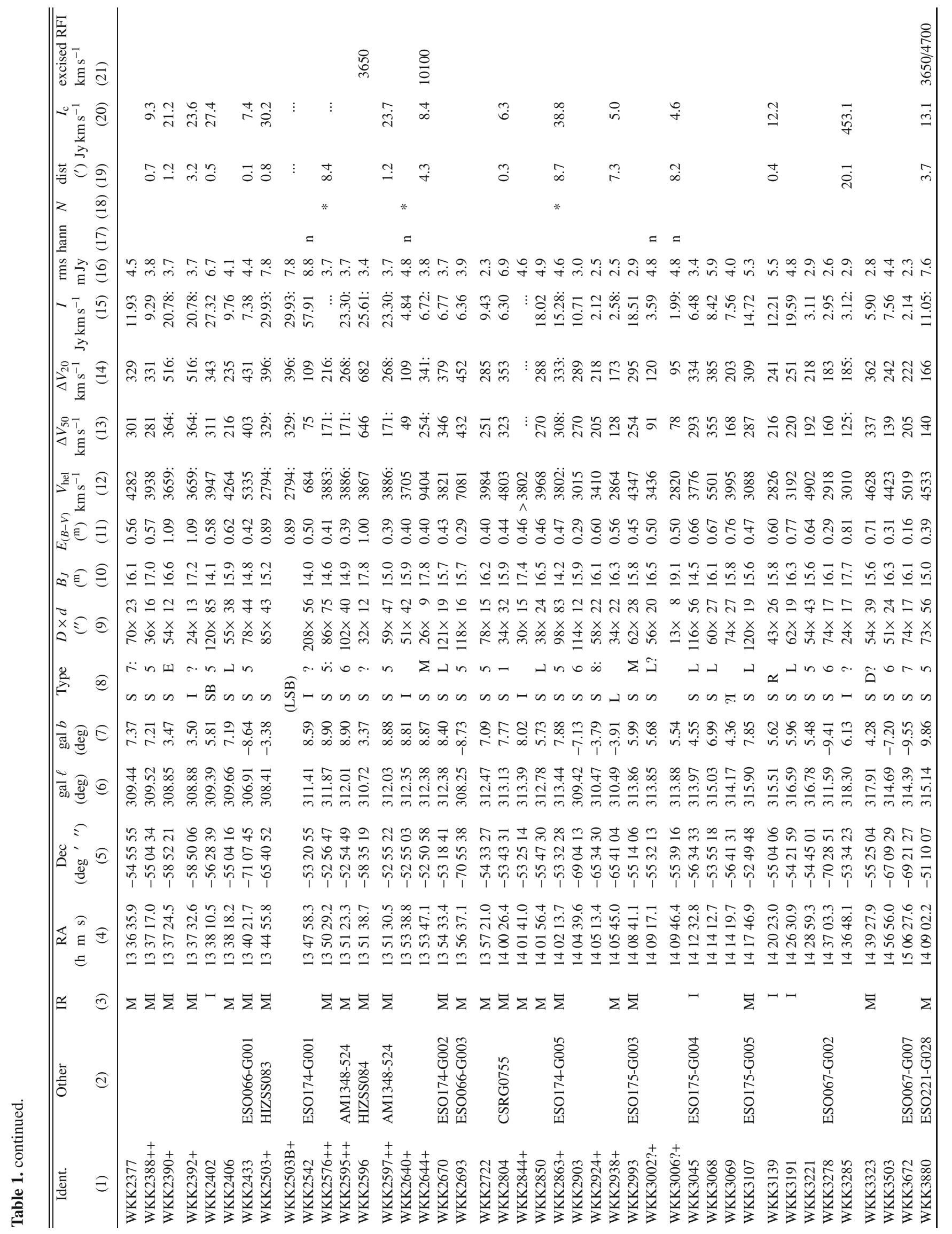




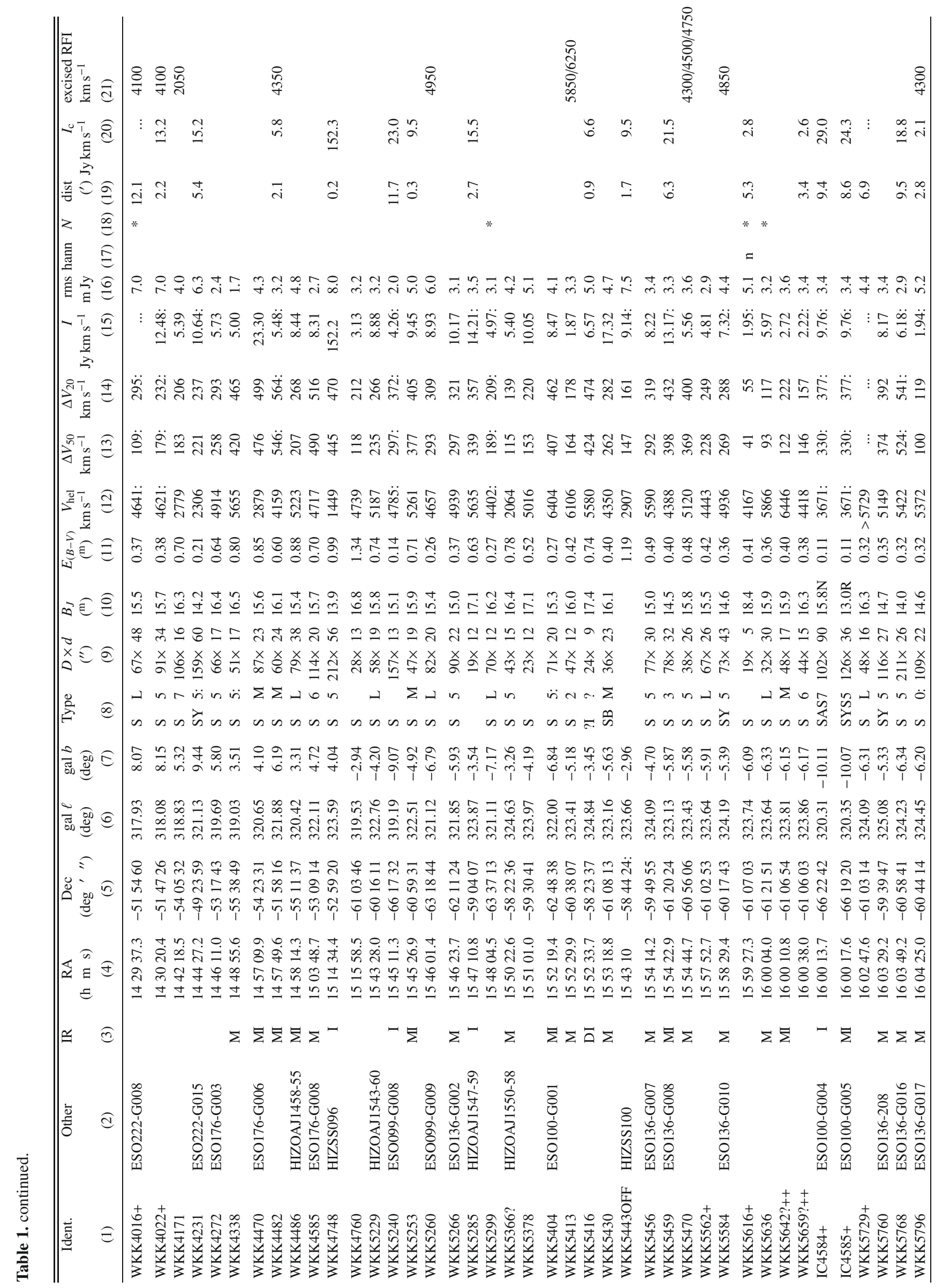




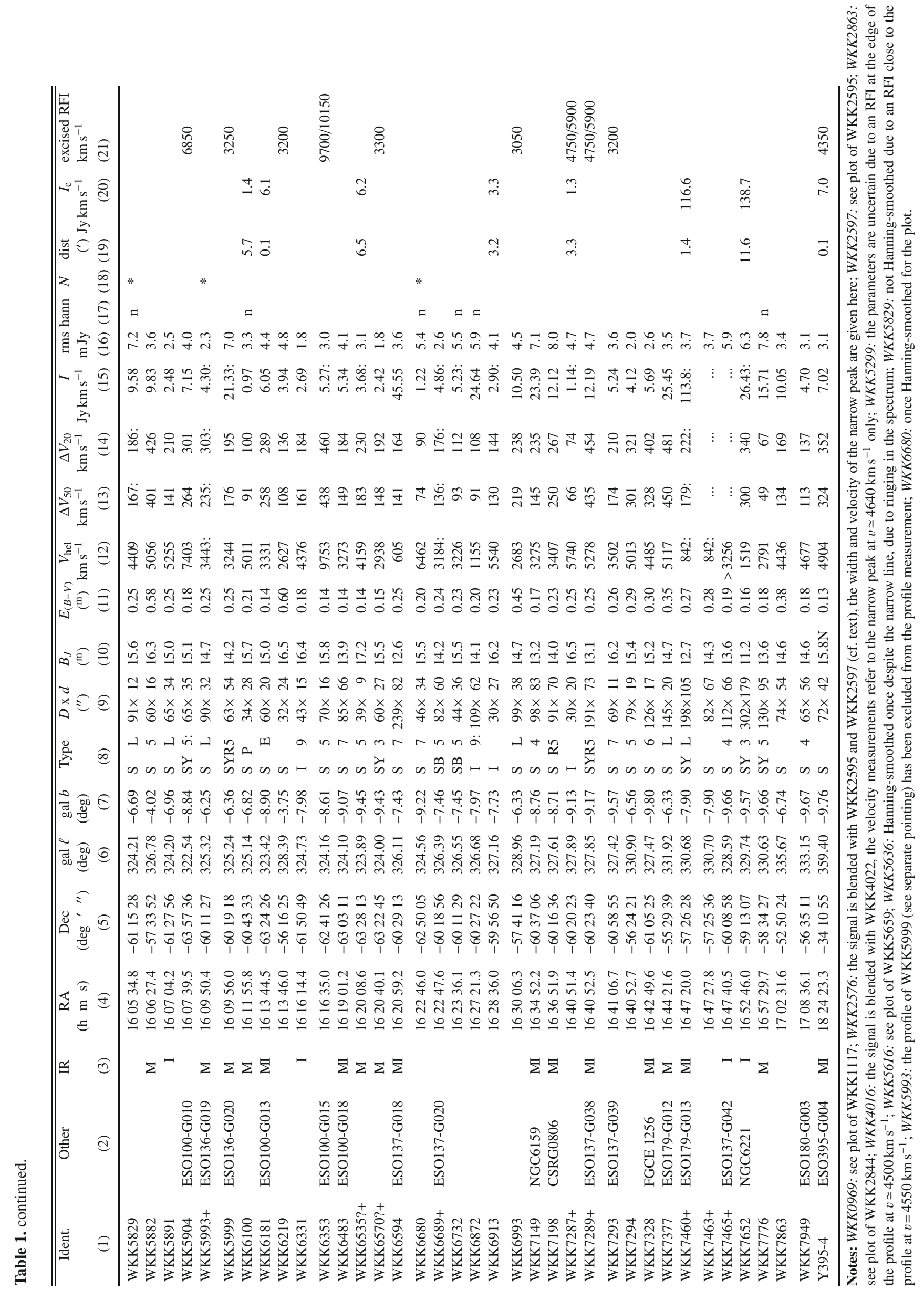


Table 2. Comparison of velocities.

\begin{tabular}{|c|c|c|c|c|c|c|c|c|c|}
\hline Ident. & $\begin{array}{r}V_{\text {hel }} \\
\mathrm{km} \mathrm{s}^{-1}\end{array}$ & $\begin{array}{c}V_{\text {other }} \\
\mathrm{km} \mathrm{s}^{-1}\end{array}$ & origin & "Reference & Ident. & $\begin{array}{r}V_{\text {hel }} \\
\mathrm{km} \mathrm{s}^{-1}\end{array}$ & $\begin{array}{c}V_{\text {other }} \\
\mathrm{km} \mathrm{s}^{-1}\end{array}$ & origin & Reference \\
\hline (1) & (2) & (3) & (4) & (5) & (1) & (2) & (3) & (4) & (5) \\
\hline WKK0204 & 4349 & $4332 \pm 8$ & HI & HIPASS & WKK3880 & 4533 & $4572 \pm 85$ & opt & RS06 \\
\hline WKK0207 & 9290 & $9178 \pm 70$ & opt & VY96 & WKK4231 & 2306 & $2254 \pm 10$ & opt \& H I & CF97 \\
\hline WKK0304 & 3813 & $3807 \pm 7$ & $\mathrm{HI}$ & HIPASS & & & $2303 \pm 6$ & $\mathrm{HI}$ & HIPASS \\
\hline WKK0491 & 7353 & $7523 \pm 250$ & opt & FW98 & WKK4272 & 4914 & $4940 \pm 9$ & $\mathrm{HI}$ & HIPASS \\
\hline WKK0539 & 7562 & $\begin{array}{r}7651 \pm 85 \\
7534+8\end{array}$ & opt & RS06 & WKK4470 & 2879 & $2875 \pm 10$ & $\mathrm{HI}$ & HIZSS \\
\hline \multirow{3}{*}{ WKK0662 } & 5603 & $\begin{array}{l}1534 \pm 8 \\
6526+231\end{array}$ & $\begin{array}{l}\text { HI } \\
\text { opt }\end{array}$ & $\begin{array}{l}\text { HIPASS } \\
\text { FW98 }\end{array}$ & & & $2877 \pm 10$ & $\mathrm{H} \mathrm{I}$ & $\begin{array}{l}\text { HIZOA } \\
\text { HIPASS }\end{array}$ \\
\hline & & $5760 \pm 76$ & opt & WK04 & WKK4486 & 5223 & $\begin{array}{lr}2890 \pm & 6 \\
5200 \pm & 10\end{array}$ & $\begin{array}{l}\mathrm{HI} \\
\mathrm{HI}\end{array}$ & $\begin{array}{l}\text { HIPASS } \\
\text { HIZOA }\end{array}$ \\
\hline & & $5630 \pm 85$ & opt & RS06 & WKK4585 & 4717 & $4728 \pm 137$ & opt & WK99 \\
\hline WKK1045 & 5411 & $5268 \pm 250$ & opt & FW98 & WKK4748 & 1449 & $1449 \pm 10$ & $\mathrm{HI}$ & HIZSS \\
\hline \multirow[t]{2}{*}{ WKK1089 } & 1712 & $3939 \pm 150$ & opt & FW98 & & & $1445 \pm 10$ & $\mathrm{HI}$ & HIZOA \\
\hline & & $1714 \pm 5$ & $\mathrm{HI}$ & HIPASS & & & $1456 \pm 3$ & H I & HIPASS-BGC \\
\hline WKK1294 & 1919 & $1910 \pm 10$ & opt \& H I & CF97 & & & $1456 \pm 5$ & $\mathrm{HI}$ & HIPASS \\
\hline WKK1352 & 5437 & $5528 \pm 100$ & opt & FW98 & WKK5229 & 5187 & $5193 \pm 10$ & $\mathrm{HI}$ & HIZOA \\
\hline WKK1446 & 3677 & $3679 \pm 9$ & $\mathrm{HI}$ & HIPASS & WKK5240 & 4785: & $4787 \pm \quad 8$ & $\mathrm{HI}$ & HIPASS \\
\hline WKK1455 & 3692 & $3684 \pm 100$ & opt & WK04 & WKK5253 & 5261 & $5093 \pm 70$ & opt & VY96 \\
\hline WKK1972 & 5651 & $5630 \pm 85$ & opt & RS06 & WKK5260 & 4657 & 4963 & opt & D91 \\
\hline \multirow{3}{*}{ WKK2029 } & 2349 & $2355 \pm 50$ & opt & FW98 & & & $4740 \pm 10$ & $\mathrm{HI}$ & HIPASS \\
\hline & & $2337 \pm 10$ & $\mathrm{HI}$ & HIZSS & WKK5266 & 4939 & 4944 & $\mathrm{HI}$ & MH91 \\
\hline & & $2340 \pm 5$ & $\mathrm{HI}$ & HIPASS & & & $4940 \pm 7$ & $\mathrm{HI}$ & HIPASS \\
\hline WKK2147 & 4180 & $4153 \pm 44$ & opt & FH95 & WKK5285 & 5635 & $5631 \pm 30$ & $\mathrm{HI}$ & HIZOA \\
\hline WKK2171 & 3709 & $3633 \pm 10$ & $\mathrm{HI}$ & HIPASS & WKK5366? & 2064 & $2059 \pm 10$ & $\mathrm{HI}$ & HIZOA \\
\hline WKK2172 & 4029 & $4069 \pm 11$ & $\mathrm{HI}$ & HIPASS & & & $4822 \pm 82$ & opt & WK04 \\
\hline WKK2222 & 2582 & $2570 \pm 7$ & $\mathrm{HI}$ & HIPASS & WKK5404 & 6404 & $6450 \pm 70$ & opt & DN97 \\
\hline \multirow[t]{3}{*}{ WKK2245 } & 2912: & $2915 \pm 10$ & $\mathrm{HI}$ & HIZSS & WKK5413 & 6106 & $6057 \pm 174$ & opt & WK99 \\
\hline & & $2903 \pm 10$ & $\mathrm{HI}$ & HIZOA & WKK5416 & 5580 & $5524 \pm 114$ & opt & WK04 \\
\hline & & $2992 \pm 85$ & opt & RS06 & & & $12403 \pm 70$ & opt & VY96 \\
\hline WKK2254 & 5518 & $5653 \pm 58$ & opt & FW06 & WKK5443OFF & 2907 & $2905 \pm 10$ & $\mathrm{HI}$ & HIZSS \\
\hline WKK2372 & 4058 & $4058 \pm 3$ & $\mathrm{HI}$ & HK01 & & & $2897 \pm 10$ & $\mathrm{HI}$ & HIZOA \\
\hline WKK2388 & 3938 & $3976 \pm 40$ & opt & FH95 & WKK5459 & 4388 & $4390 \pm 60$ & opt & $\mathrm{RC} 3$ \\
\hline \multirow[t]{2}{*}{ WKK2390 } & 3659: & $3790 \pm 70$ & opt & VY96 & & & $4396 \pm 7$ & $\mathrm{HI}$ & HIPASS \\
\hline & & $3586 \pm 10$ & $\mathrm{HI}$ & HIPASS & WKK5470 & 5120 & $5209 \pm 214$ & opt & WK99 \\
\hline WKK2392 & 3659: & $3790 \pm 70$ & opt & VY96 & WKK5584 & 4936 & 5027 & opt & D91 \\
\hline WKK2402 & 3947 & $3956 \pm 6$ & $\mathrm{HI}$ & HIPASS & WKK5642? & 6446 & $6045 \pm 42$ & opt & SH92 \\
\hline WKK2433 & 5335 & $5367 \pm 53$ & opt & FH95 & & & $6118 \pm 100$ & opt & WK04 \\
\hline WKK2503? & 2794: & $2789 \pm 10$ & $\mathrm{HI}$ & HIZSS & IC4584 & 3671: & $3700 \pm 44$ & opt & SH92 \\
\hline & & $2769 \pm 10$ & $\mathrm{HI}$ & HIZOA & IC4585 & 3671: & $3638 \pm 40$ & opt & RC3 \\
\hline & & $2774 \pm 6$ & $\mathrm{HI}$ & HIPASS & WKK5768 & 5422 & $5426 \pm 10$ & $\mathrm{HI}$ & $\mathrm{RC} 3$ \\
\hline WKK2542 & 684 & $694 \pm 6$ & $\mathrm{HI}$ & HK01 & & & $5428 \pm 6$ & $\mathrm{HI}$ & HIPASS \\
\hline & & 680 & HI & BD99 & WKK5796 & 5372 & $5260 \pm 60$ & opt & $\mathrm{RC} 3$ \\
\hline & & $688 \pm 3$ & $\mathrm{HI}$ & HIPASS-BGC & WKK5993 & 3443: & $3487 \pm 108$ & opt & WK04 \\
\hline & & $687 \pm 5$ & $\mathrm{HI}$ & HIPASS & WKK5999 & 3244 & $3250 \pm 38$ & opt & WK04 \\
\hline WKK2576 & 3883: & $3948 \pm 70$ & opt & DN97 & & & $3246 \pm 6$ & $\mathrm{HI}$ & HIPASS \\
\hline & & $3876 \pm 85$ & opt & RS06 & WKK6181 & 3331 & $3278 \pm 70$ & opt & VY96 \\
\hline & & $3872 \pm 5$ & $\mathrm{HI}$ & HIPASS & & & $3308 \pm 70$ & opt & WK99 \\
\hline WKK2595 & 3886: & $3873 \pm 85$ & opt & RS06 & WKK6353 & 9753 & $9900 \pm 70$ & opt & DN97 \\
\hline WKK2596 & 3867 & $3869 \pm 10$ & $\mathrm{HI}$ & HIZSS & WKK6483 & 3273 & 3367 & opt & D91 \\
\hline & & $3848 \pm 10$ & $\mathrm{HI}$ & HIZOA & & & $3268 \pm 17$ & $\mathrm{HI}$ & HIPASS \\
\hline & & $3881 \pm 7$ & $\mathrm{HI}$ & HIPASS & WKK6594 & 605 & $606 \pm 20$ & opt & HG95 \\
\hline WKK2597 & 3886: & $3973 \pm 43$ & opt & SH92 & & & $605 \pm \quad 3$ & $\mathrm{HI}$ & HIPASS-BGC \\
\hline & & $3954 \pm 85$ & opt & RS06 & & & $605 \pm$ & $\mathrm{HI}$ & HIPASS \\
\hline WKK2640 & 3705 & $3574 \pm 85$ & opt & RS06 & WKK6689 & 3184: & $3239 \pm 88$ & opt & WK04 \\
\hline WKK2644 & 9404 & $9406 \pm 100$ & opt & WK04 & WKK6872 & 1155 & $1157 \pm 3$ & $\mathrm{HI}$ & HIPASS-BGC \\
\hline & & $9276 \pm 85$ & opt & RS06 & & & $1157 \pm 5$ & $\mathrm{HI}$ & HIPASS \\
\hline WKK2670 & 3821 & $3758 \pm 40$ & opt & FH95 & WKK7149 & 3275 & $3300 \pm 30$ & opt & SE95 \\
\hline & & $3798 \pm 85$ & opt & RS06 & & & $3263 \pm 6$ & $\mathrm{HI}$ & HIPASS \\
\hline WKK2693 & 7081 & $7389 \pm 250$ & opt & FW98 & WKK7198 & 3407 & $3405 \pm 6$ & $\mathrm{HI}$ & HIPASS \\
\hline & & $7013 \pm 80$ & opt & WK04 & WKK7289 & 5278 & $2100 \pm 100$ & opt & F83 \\
\hline WKK2804 & 4803 & $4779 \pm 39$ & opt & FH95 & WKK7377 & 5127 & $5122 \pm 7$ & $\mathrm{HI}$ & HIPASS \\
\hline WKK2850 & 3968 & $3959 \pm 6$ & $\mathrm{HI}$ & HIPASS & WKK7460 & 842: & $775 \pm 36$ & opt & SH92 \\
\hline WKK2863 & 3802: & $3775 \pm 37$ & opt & SH92 & & & $842 \pm \quad 4$ & $\mathrm{HI}$ & HIPASS-BGC \\
\hline & & $3778 \pm 30$ & opt & SE95 & & & $843 \pm$ & $\mathrm{HI}$ & HIPASS \\
\hline & & $3768 \pm 6$ & $\mathrm{HI}$ & HIPASS & WKK7465 & $>3256$ & $3255 \pm 39$ & opt & SH92 \\
\hline WKK2938 & 2864 & $3024 \pm 157$ & opt & FW98 & & & $3265 \pm 4$ & $\mathrm{HI}$ & DN96 \\
\hline WKK2993 & 4347 & $4313 \pm 37$ & opt & FH95 & & & $3283 \pm 85$ & opt & RS06 \\
\hline & & $4364 \pm 45$ & opt & FW98 & WKK7652 & 1519 & $1350 \pm 31$ & opt & RC3 \\
\hline & & $4345 \pm 7$ & $\mathrm{HI}$ & HIPASS & & & $1478 \pm 38$ & opt & WK04 \\
\hline WKK3107 & 3088 & $3090 \pm 7$ & $\mathrm{HI}$ & HIPASS & & & $1340 \pm 85$ & opt & RS06 \\
\hline WKK3139 & 2826 & $2844 \pm 70$ & opt & VY96 & & & $1499 \pm 5$ & $\mathrm{HI}$ & HIPASS \\
\hline & & $2823 \pm 7$ & $\mathrm{HI}$ & HIPASS & WKK7776 & 2791 & $2790 \pm 3$ & $\mathrm{HI}$ & HIPASS-BGC \\
\hline WKK3191 & 3192 & $3187 \pm 6$ & $\mathrm{HI}$ & HIPAS & & & $2790 \pm 5$ & $\mathrm{HI}$ & HIPASS \\
\hline WKK3278 & 2918 & $3118 \pm 40$ & opt & FW98 & Y395-4 & 4904 & $4896 \pm 43$ & opt & FH95 \\
\hline WKK3285 & 3010 & 3016 & $\mathrm{HI}$ & RK02 & & & $4895 \pm 9$ & $\mathrm{HI}$ & TH07 \\
\hline & & $3016 \pm 3$ & $\mathrm{HI}$ & HIPASS-BGC & & & & & \\
\hline & & $3017 \pm 5$ & HI & HIPASS & & & & & \\
\hline
\end{tabular}




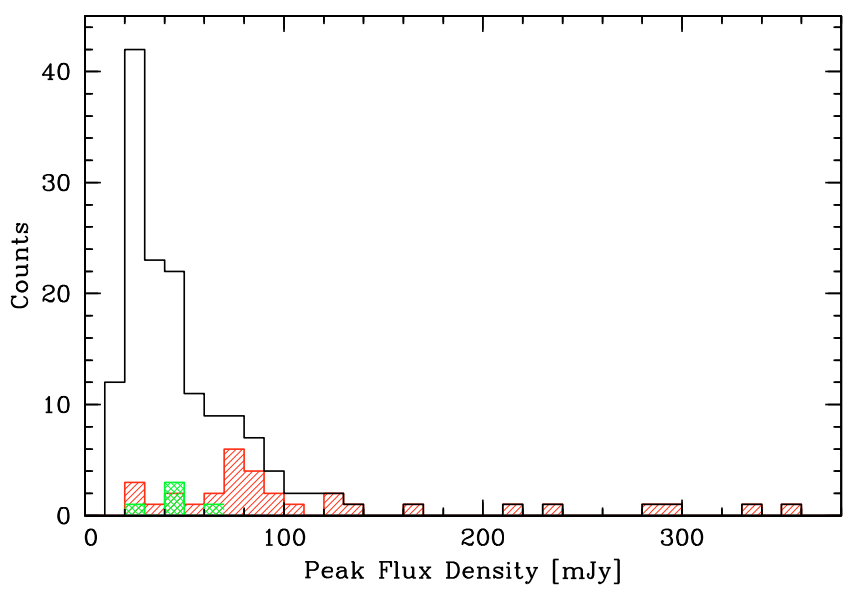

Fig. 2. Histogram of peak flux density in mJy of our detections (open) and of the detections in common with HIPASS (hashed). Cross-hashed detections are HIPASS detections with errors $\geq 10 \mathrm{~km} \mathrm{~s}^{-1}$. Note that there are three more detections by both surveys beyond the frame of the plot between $400 \mathrm{mJy}$ and $800 \mathrm{mJy}$.

with HIPASS confirms that corrections for distances up to half the beam width (i.e., 7.5) are acceptable.

\section{The non-detections}

A further 152 galaxies that were observed in the Crux and GA regions were not detected. They are listed in Table 4 with the searched velocity range as well as the rms within that interval. Some spectra did reveal a signal, but careful investigation showed them to be due to close neighbours rather than the targeted galaxies. These cases are marked with a plus in Col. 1 of Table 4 (see also Table 1), and the identification of the signal is given in the footnote. Some of these cases are discussed in detail in Appendix A.

Columns 1-11: same as in Table 1. CGMW4\# in Col. 2 stands for the 4th Catalog of Galaxies Behind the Milky Way (Roman et al. 2000).

Column 12: the searched velocity range in $\mathrm{km} \mathrm{s}^{-1}$.

Column 13: the rms noise of the searched velocity range in mJy, typically of the order of $4 \mathrm{mJy}$. These values were determined after baseline fitting over a width of $1600 \mathrm{~km} \mathrm{~s}^{-1}$ - hence similar to the determination for detections - centred at increasingly higher redshifts in order to obtain values for the whole velocity range. The quoted values represent the highest rms for the velocity intervals, the rms for the nearer velocities are on average slightly lower.

Column 14: perturbed velocity intervals, mainly due to recurring RFI around 800, 1250 and $\sim 7400 \mathrm{~km} \mathrm{~s}^{-1}$, very strong GPS (Global Positioning System) signals around 8300-8500 $\mathrm{km} \mathrm{s}^{-1}$, and detections of other galaxies. In these intervals a signal would have gone undetected.

Column 15: a star indicates a footnote for this entry.

Column 16: the distance in arcminutes of the targeted galaxy to the centre of the beam. Given the falloff of sensitivity with distance from the centre of the beam, any upper limits for flux densities calculated from the rms of such cases are underestimated.

Columns 17-19: independent velocity determinations and error for the non-detected galaxies. The reference coding (Col. 18) is as in Table 3, and Col. 19 gives the origin of the measurement (optical or $\mathrm{HI}$ ).
Table 3. References for independent velocity determinations.

\begin{tabular}{ll}
\hline \hline BD99: & Banks et al. (1999) \\
CF97: & Coté et al. (1997) \\
DN96: & Di Nella et al. (1996) \\
DN97: & Di Nella et al. (1997) \\
DT90: & Djorgovski et al. (1990) \\
D91: & Dressler (1991) \\
F83: & Fairall (1983) \\
FW98: & Fairall et al. (1998) \\
FW06: & Fairall \& Woudt (2006) \\
FH95: & Fisher et al. (1995) \\
HW00: & Hasegawa et al. (2000) \\
HIZSS: & Henning et al. (2000) \\
HIPASS: & HIPASS (2006) \\
HG95: & Huchra et al. (1995) \\
HK01: & Huchtmeier et al. (2001) \\
JS00: & Juraszek et al. (2000) \\
KD04: & Koribalski \& Dickey (2004) \\
HIPASS-BGC & Koribalski et al. (2004) \\
MF96: & Mathewson \& Ford (1996) \\
MH91: & Mould et al. (1991) \\
PT03: & Paturel et al. (2003) \\
RS06: & Radburn-Smith et al. (2006) \\
RK02: & Ryan-Weber et al. (2002) \\
SE95: & Sanders et al. (1995) \\
SH92: & Strauss et al. (1992) \\
TH07: & Theureau et al. (2007) \\
RC3: & de Vaucouleurs et al. (1991) \\
Vv92: & Visvanathan \& van den Bergh (1992) \\
VY96: & Visvanathan \& Yamada (1996) \\
WK99: & Woudt et al. (1999) \\
WK04: & Woudt et al. (2004) \\
\hline
\end{tabular}

Based on this table, only $42 \%$ of the 149 pointings have an unperturbed velocity range, and $52 \%$ of the pointings are RFI free (i.e., 18 pointings are affected by the detection of a galaxy, either in the ON or in the OFF scan). Table 5 lists the frequencies of the noted velocity ranges affected by RFIs which affect the possible detection of a galaxy. Note that the single 10-min scans show many more RFIs, most of which, however, could be excised successfully. The worst affected velocity ranges are either very low ( $800-1350 \mathrm{~km} \mathrm{~s}^{-1}$ for $23 \%$ of pointings) or around $8300-8500 \mathrm{~km} \mathrm{~s}^{-1}$ for $14 \%$ of the cases. In $8 \%$ of the cases the velocity range $7000-7700 \mathrm{~km} \mathrm{~s}^{-1}$ shows some problems.

WKK 3836 is the only galaxy that we have not detected but was subsequently detected by HIPASS. On the one hand, our rms of $7 \mathrm{mJy}$ is fairly high (only $13 \%$ of our detection have an rms of $7 \mathrm{mJy}$ and higher) and the pointing is $d=8.5$ away from the galaxy position (which means the signal would be reduced by a factor of 2.4). On the other hand, the HIPASS detection is weak (the peak flux density is $37 \mathrm{mJy}$ and the error on the velocity at $12 \mathrm{~km} \mathrm{~s}^{-1}$ is the second highest value in our sample of HIPASS galaxies).

\section{Velocity distribution and detection rate}

Figure 3 shows the Crux/GA search area (outlined regions) in Galactic coordinates with the optically discovered galaxies $(D \lesssim 0.2)$ plotted as small dots (Woudt \& Kraan-Korteweg 2001). The 314 galaxies observed with the Parkes radio telescope (indicated with larger symbols) are distributed fairly homogeneously over the galaxy density distribution - leading naturally to a larger number of observations in the high density area 


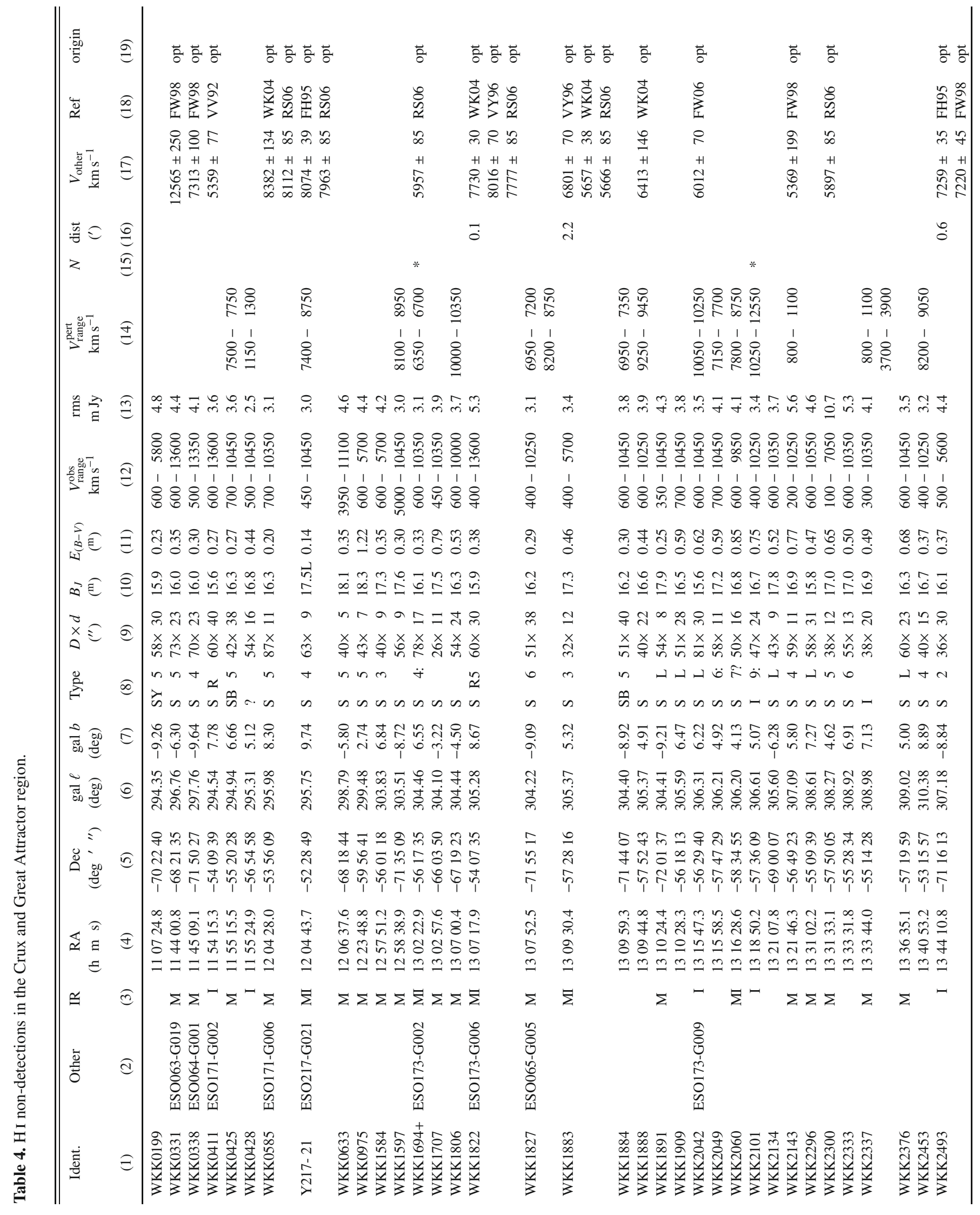




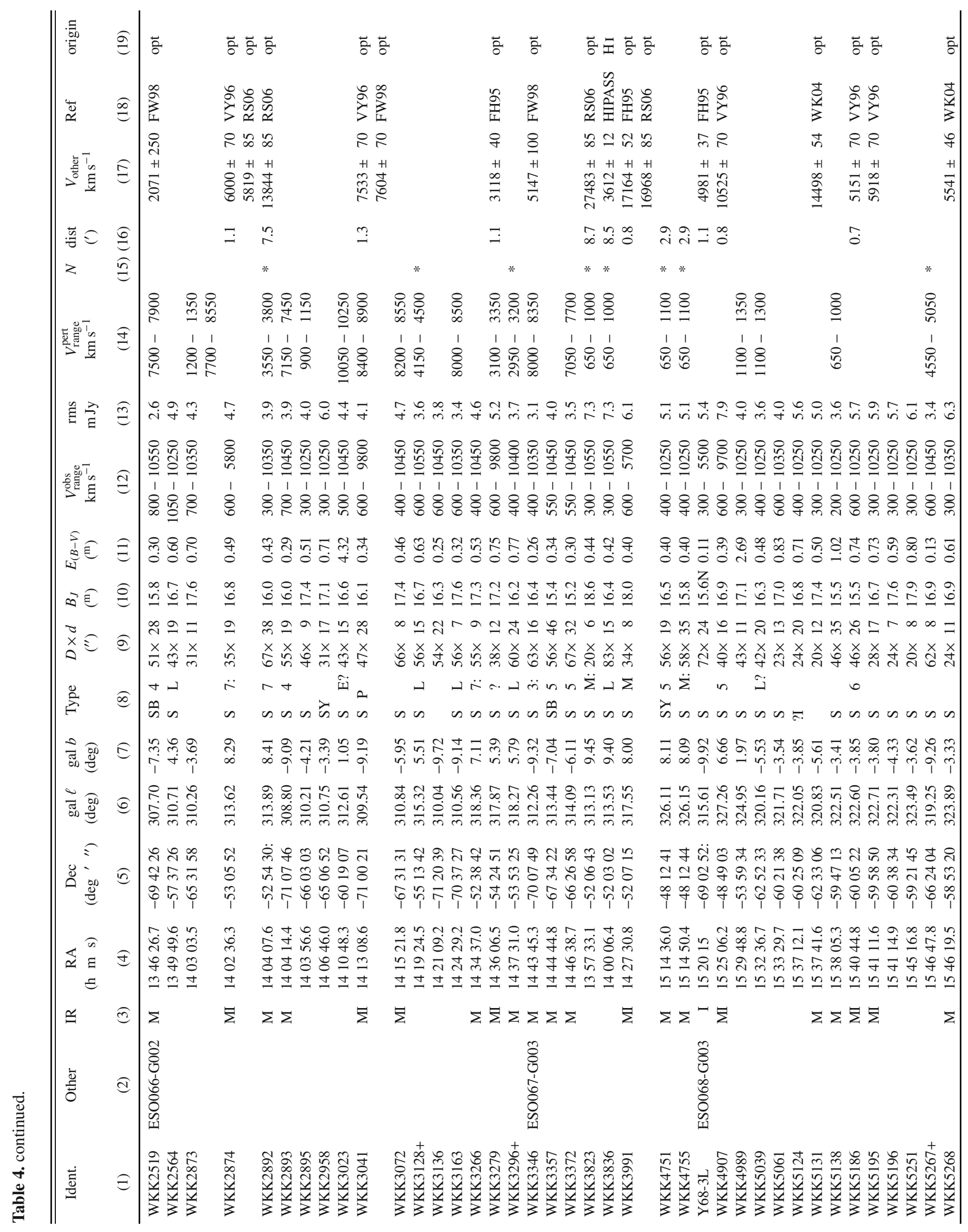




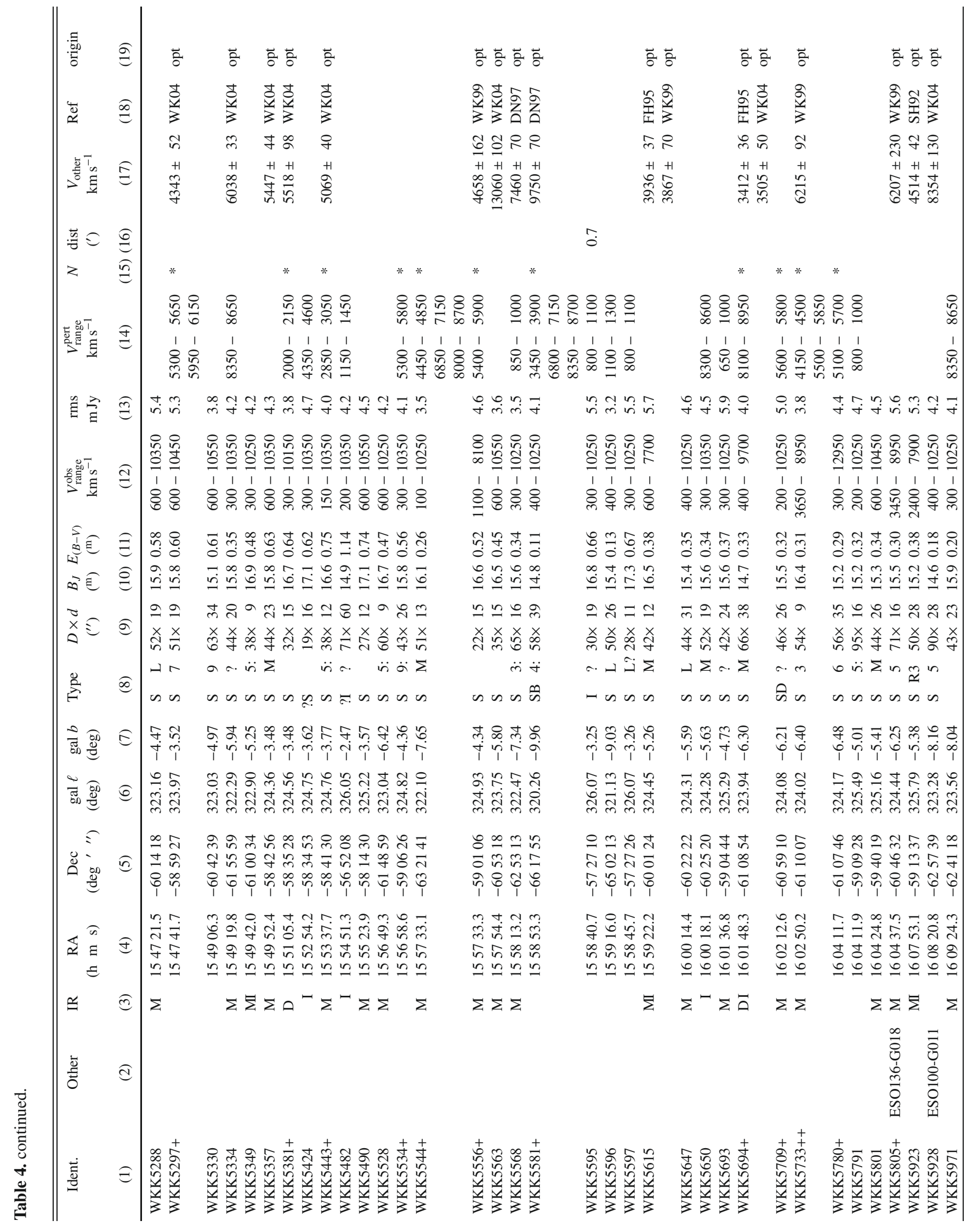




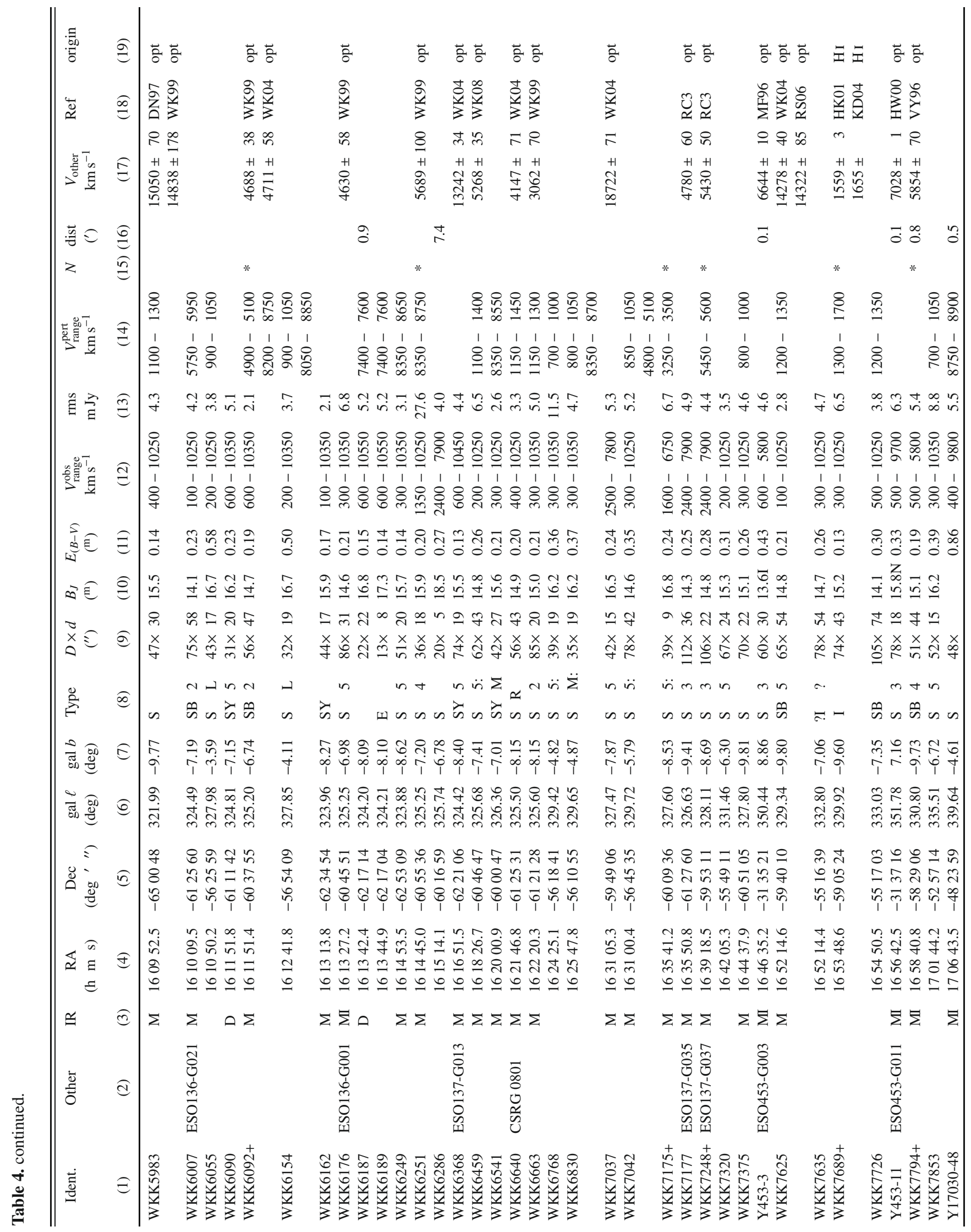


of the Norma cluster. The contours mark extinction levels of $A_{B}={ }^{\mathrm{m}} .0$ and 3.0 following Schlegel et al. (1998). The former indicates where previous optical surveys become incomplete, the latter where the deep optical ZOA survey becomes incomplete (Kraan-Korteweg 2000; Woudt \& Kraan-Korteweg 2001). Detected galaxies are marked by filled circles, non-detected galaxies by crossed squares. The detections are colour-coded for radial velocity in such a way that they emphasise the velocity space of the GA region (blue dots; $3000<v_{\text {hel }}<6500 \mathrm{~km} \mathrm{~s}^{-1}$ ), showing the Norma cluster and Norma Wall that (is believed to) encompass the GA overdensity. Magenta dots are at lower velocities, while the red dots indicate higher velocities.

The majority of the detections are found to lie in the velocity range of the GA overdensity $\left(3000<v_{\text {hel }}<6500 \mathrm{~km} \mathrm{~s}^{-1}\right)$. Few nearer galaxies are detected $\left(V_{\text {hel }}<3000 \mathrm{~km} \mathrm{~s}^{-1}\right)$, even though we are sensitive to them. And very few more distant galaxies were detected. This is partly due to the lower sensitivity at these higher redshifts, but also indicative of the lack of distant largescale structures in this area.

This is further illustrated by a histogram of the heliocentric radial velocities for the detections presented in this paper (Fig. 4). The overall histogram shows all detections. Detections in the GA survey region are marked in blue, and the ones in the Crux survey region in red. The inset shows the velocity histograms for the Crux and GA region separately, including the previous work in the adjacent Hydra/Antlia survey region.

The histogram shows a markedly different behaviour from what is expected for a uniform distribution of galaxies in space given our rms, with a sharp drop-off at about $6000 \mathrm{~km} \mathrm{~s}^{-1}$. The peak - albeit quite broad - is centred roughly at $4500 \mathrm{~km} \mathrm{~s}^{-1}$ ranging from $3000-6000 \mathrm{~km} \mathrm{~s}^{-1}$, similar to the velocity range of the Norma cluster (Woudt et al. 2008) which completely coincides with the predicted velocity range of the GA (Kolatt et al. 1995) and the mean Norma cluster velocity (Woudt et al. 2008). It is distinct from the much flatter distribution of the H I-survey undertaken under the same observing conditions for the Hydra/Antlia survey area (top panel of inset; as in Paper I).

This is in agreement with the results by Woudt et al. (2004) who have shown that the velocity distribution of all survey galaxies for which we obtained redshifts (about 15\% on average for the deep ZOA surveys, mostly optical spectra from our dedicated follow-up surveys at the SAAO and ESO, plus some previously published redshifts), is fairly flat out to $20000 \mathrm{~km} \mathrm{~s}^{-1}$ in the Hydra/Antlia region, while the Crux region and - much more pronounced - the GA region show a distinct broad peak of galaxies at $\sim 4000-5000 \mathrm{~km} \mathrm{~s}^{-1}$ (see their Fig. 5), while the histogram is otherwise similar for the three survey regions. The prominence of the GA overdensity around $\ell \sim 320^{\circ}$ leads to an overall higher fraction of nearby galaxies $\left(V \lesssim 6000 \mathrm{~km} \mathrm{~s}^{-1}\right)$ over the sampled volume (out to about $20000 \mathrm{~km} \mathrm{~s}^{-1}$ ). This may well explain the slightly higher detection rate of $52 \%$ found for this survey compared to Paper I (45\%, with a total 148 observed galaxies compared to 314 here). In general, the non-detections are for fainter and smaller galaxies (extinction-corrected), implying that they are most likely background galaxies beyond the velocity search range of our survey, plus some closer-by galaxies with an H I flux density below our sensitivity limit.

Inspection of Fig. 3 indicates that the detections and nondetections are spread quite evenly with respect to the galaxy density of the overall galaxy distribution. We subsequently have a larger number of observations in and around the high density area of the Norma cluster. A relatively higher fraction of observations were also made at higher extinction levels: (i) in the suspected extension of the Norma Wall across the Galactic plane, 


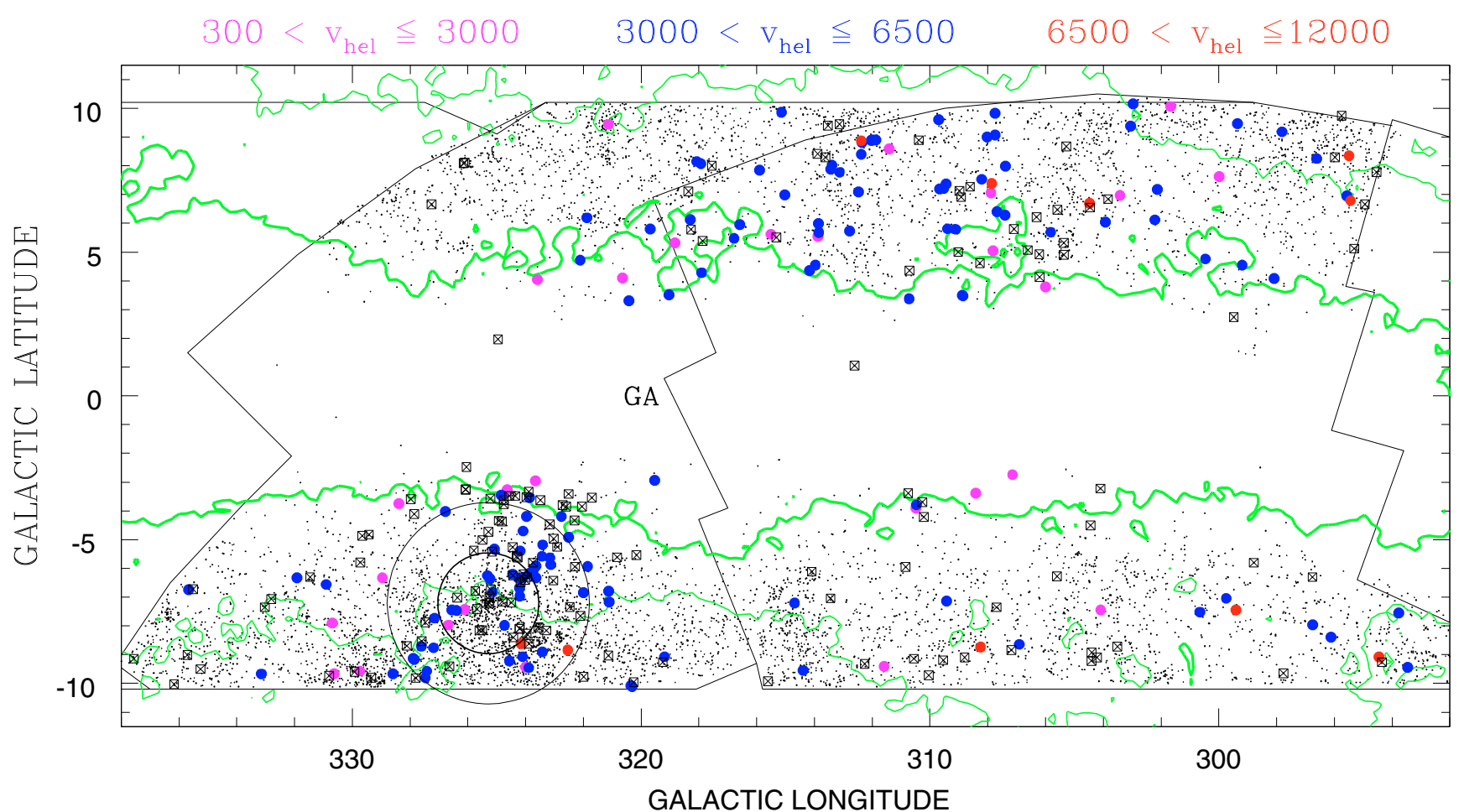

Fig. 3. Distribution of galaxies in the Crux and GA ZOA regions. Filled circles indicate the $16221 \mathrm{~cm}$ detections, crosses in squares the $152 \mathrm{mon}-$ detections. The small dots represent the galaxies uncovered within the optical search regions (outlined areas). The contours show the dust extinction as determined from the $100 \mu \mathrm{m}$ DIRBE maps (Schlegel et al. 1998) at the levels $A_{B}=1.0$ and 3. 0 (thick line). The circles are centred on the Norma cluster with $1 R_{\mathrm{A}}$ and $2 R_{\mathrm{A}}$.

i.e., extending from the Norma cluster at $(\ell, b, v)=\left(325.29^{\circ}\right.$, $-7.21^{\circ}, 4821 \mathrm{~km} \mathrm{~s}^{-1}$ ) (Woudt et al. 2008) towards the lowlatitude CIZA J1324.7-5736 and Cen-Crux clusters at $(\ell, b, v)=$ $\left(307.4^{\circ},+5.0^{\circ}, 5700 \mathrm{~km} \mathrm{~s}^{-1}\right)$ and $\left(305^{\circ},+5^{\circ}, 6214 \mathrm{~km} \mathrm{~s}^{-1}\right)$, respectively (Radburn-Smith et al. 2006, see also their Fig. 4), which form part of the GA Wall; (ii) for some highly obscured galaxy candidates deep into the ZOA (in total 37 were observed with $A_{B}>3$. 0 of which 20 were detected) as $\mathrm{H}$ I observations are the only way to obtain a redshift for such heavily obscured galaxies.

Overall the detections and non-detections seem to be similarly distributed over the survey area. However, a closer look at the Norma cluster (see also Fig. 5) reveals that the detection rate within one Abell radius $\left(R_{\mathrm{A}}=1.75\right)$ of the central cD galaxy, WKK 6292, is only $41 \%\left(n_{\text {tot }}=32\right)$, which is lower than for the whole survey on the $1 \sigma$ level. A bit further out, in the annulus $1-2 R_{\mathrm{A}}$, the detection rate is similar to the rest of the GA/Crux survey, namely $53 \%\left(n_{\text {tot }}=53\right)$. Such a trend, if real, would be expected if there are not many spiral galaxies in the cluster or if the spirals are HI deficient. The former is unlikely as morphological distinction between ellipticals and spirals is largely unaffected at the extinction levels of the Norma cluster. The latter is probable since rich, massive, and X-ray strong galaxy clusters like the Norma cluster generally show H I deficiencies (Giovanelli \& Haynes 1985). We explore this effect in more detail in Sect. 6.

\section{HI deficiency in the Norma cluster}

As a first measure of HI-deficiency we regard the HI-masses of the galaxies in and around the cluster as a function of
Table 5. References for independent velocity determinations.

\begin{tabular}{rr}
\hline \hline Velocity range & Counts \\
\hline $800-1000$ & 19 \\
$1200-1350$ & 15 \\
3200 & 1 \\
$3700-3800$ & 2 \\
4450 & 2 \\
4900 & 1 \\
5800 & 1 \\
6000 & 1 \\
7000 & 3 \\
$7200-7300$ & 3 \\
$7500-7700$ & 6 \\
8100 & 1 \\
$8300-8500$ & 21 \\
$8800-9000$ & 2 \\
9300 & 1 \\
10000 & 1 \\
$10100-10300$ & 4 \\
\hline
\end{tabular}

cluster-centric distance. The HI masses are calculated using $M_{\mathrm{HI}}=2.356 \times 10^{5} D^{2} S$, where $S$ is the H I flux integral in $\mathrm{Jy} \mathrm{km} \mathrm{s}{ }^{-1}$, and $D$ the distance in Mpc calculated from the measured velocity and corrected for the motion with respect to the Local Group (Yahil et al. 1977). For galaxies assumed to lie in the Norma cluster (i.e., within $1.5 R_{\mathrm{A}}$ and $2096 \mathrm{~km} \mathrm{~s}^{-1}<v<$ $7646 \mathrm{~km} \mathrm{~s}^{-1}$, cf. Woudt et al. 2008) we adopt a distance of $D=67 h_{70}^{-1} \mathrm{Mpc}$.

Figure 6 shows histograms of the logarithm of H I mass for galaxies in the inner region of the Norma cluster $\left(<0.5 R_{\mathrm{A}}\right.$, top panel), three different annuli $\left(0.5-1 R_{\mathrm{A}} ; 1-1.5 R_{\mathrm{A}}\right.$, 


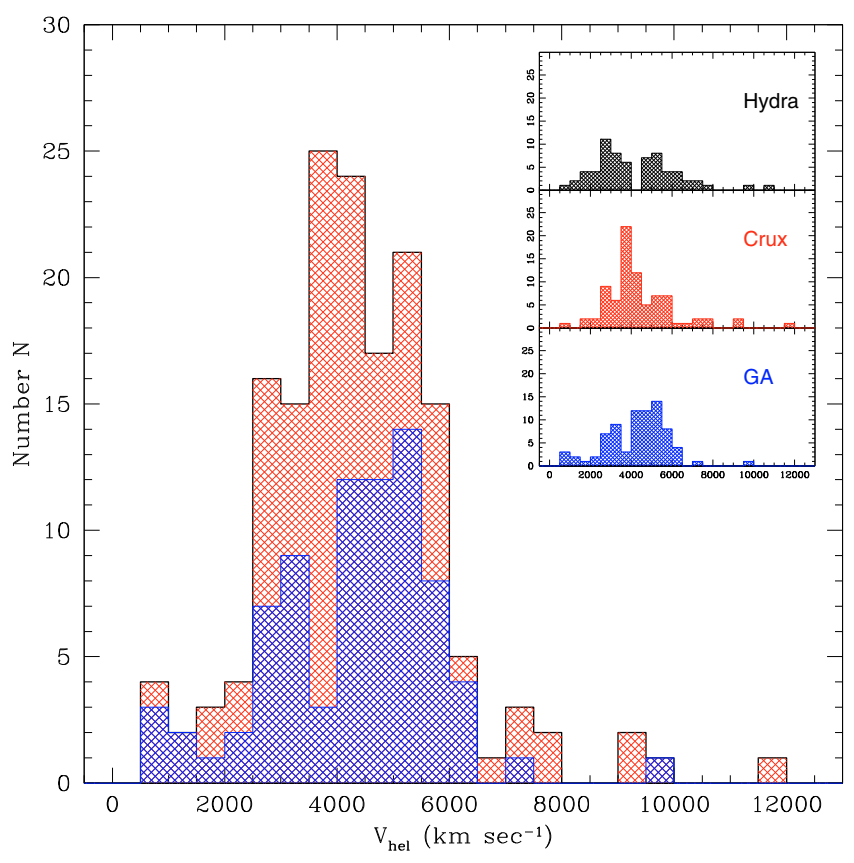

Fig. 4. Velocity distribution of the $162 \mathrm{HI}$ detections in the Crux (light grey/red) and GA (dark grey/blue) regions. The inset shows the detections separated by regions; the detections in the Hydra/Antlia region from Paper I are also shown.

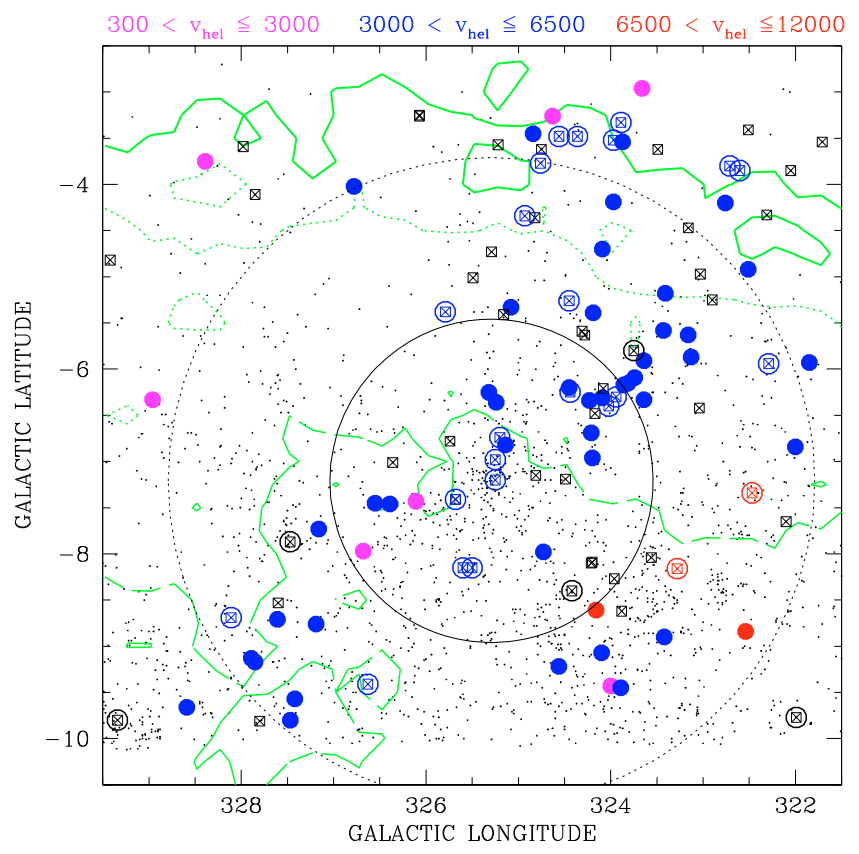

Fig. 5. Distribution of the galaxies observed at $21 \mathrm{~cm}$ zoomed in at the Norma cluster (cf. description of Fig. 3). A third contour level at $A_{B}=$ 2 . 0 is shown as a dotted line. Non-detections with a large circle have a known optical velocity (a black circle stands for $v>12000 \mathrm{~km} \mathrm{~s}^{-1}$ ).

and 1.5-2 $R_{\mathrm{A}}$, middle panels), including for comparison the $\mathrm{H}$ I-mass distribution of all the 162 detections in the survey area (bottom panel). For reference, the hashed histograms are shown in accumulation in the bottom panel as well. The mean values and the standard deviations are indicated for each subset and for the total region in the bottom panel. Our median value of all detections is $\log M_{\mathrm{HI}}=9.86$ (in units of solar mass) and compares well with the H I mass distribution in the Northern Extension of

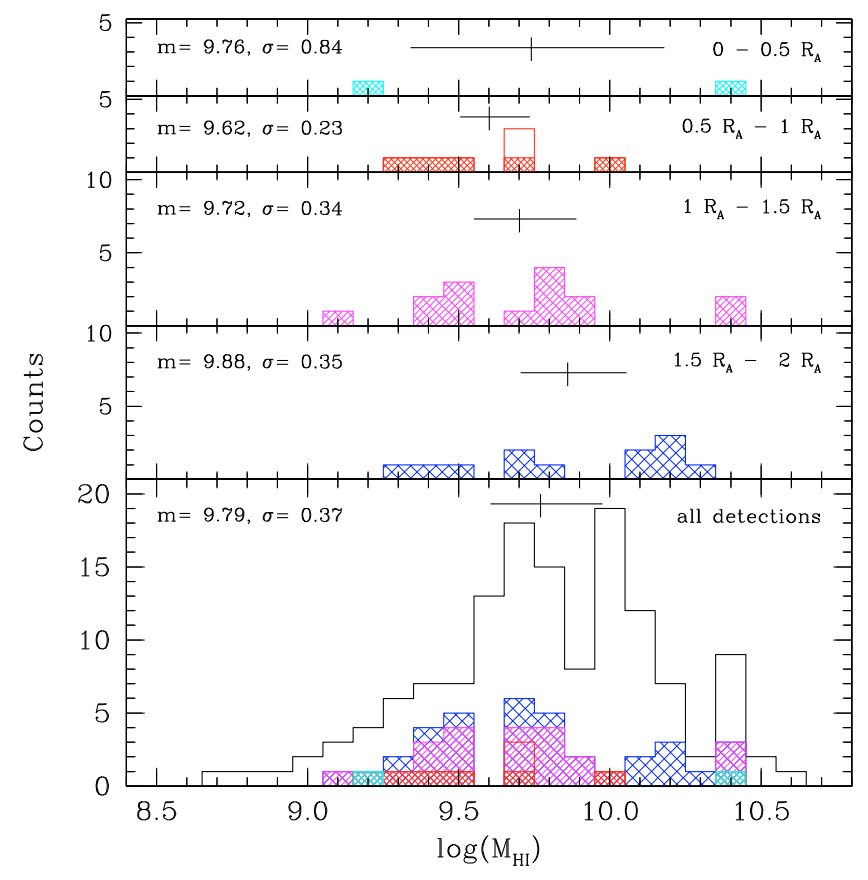

Fig. 6. Histogram of the logarithm of H I mass for various regions in the Norma cluster (hashed) and the total region observed (non-hashed). The non-hashed region in the second panel from the top indicates two galaxies (WKK 6689 and WKK 6732) with an uncertain though small contribution from each other. The H I mass in both cases is therefore slightly overestimated.

the HIZOA survey $\left(\log M_{\mathrm{HI}}=9.7\right.$, Donley et al. 2005$)$ which has s similar sensitivity to our pointed survey. These histograms show that galaxies closer to the cluster core have lower H I mass than farther galaxies, although at only about the $1 \sigma$ level.

In the innermost region only two galaxies (out of nine; 22\%) were detected, one of which actually has a substantial H I-mass (WKK 5999 lies at $d=0.49 R_{\mathrm{A}}$ and $v=3244 \mathrm{~km} \mathrm{~s}^{-1}$ ). Apart from this galaxy, the distribution of H I mass for the galaxies within $1.5 R_{\mathrm{A}}$ is shifted towards the lower end as compared to the field. This is expected if we assume that cluster members have passed at least once through the centre of the cluster and have undergone ram-pressure stripping. In contrast, galaxies in the range $1.5-2 R_{\mathrm{A}}$ have an $\mathrm{H}$ I mass distribution comparable to the field.

A better estimate of the effect of the cluster environment on the H I content of the Norma spiral galaxies is the H I deficiency parameter (Giovanelli \& Haynes 1985) which compares the H I content of a cluster galaxy with the average H I content of an isolated field spiral of the same morphological type. Solanes et al. (1996) have shown that the H I content of a field spiral also depends on the diameter. However, due to the uncertainties in diameter of our highly obscured galaxies we have not included the diameter dependence in our calculations. Moreover, there is uncertainty in the morphology of many of the highly obscured galaxies. Such obscured galaxies were generally labelled as "S" by Woudt \& Kraan-Korteweg (2001) without a further subtype. We assumed these to be late type spirals (i.e., Scd or Sd,) as a bulge dominated spiral could have been classified, whereas strongly obscured low-surface brightness irregular galaxies would most likely have remained invisible on the optical survey plate.

We note furthermore, that our sample of latest type spirals $(\mathrm{Sdm}-\mathrm{I})$ shows a systematic offset in H I mass compared 
to our other morphological sub-samples. The mean H I mass of this latest type sample (not including the possibly $\mathrm{H}$ I-deficient Norma galaxies within $2 R_{\mathrm{A}}$ ) is $\log M_{\mathrm{HI}}=9.79$ with a standard deviation of 0.33 . This value is typical of Scd and $\mathrm{Sd}$ galaxies $\left(\log M_{\mathrm{HI}}=9.62\right.$, with a standard deviation of 0.31$)$ and consistent with the fact that Giovanelli \& Haynes (1985) find the value 9.09 for their field $\mathrm{Sm}$ - Im sample (all H I masses from Giovanelli \& Haynes were corrected for the difference of their adopted Hubble constant of $50 \mathrm{~km} \mathrm{~s}^{-1} \mathrm{Mpc}^{-1}$ to our value of $70 \mathrm{~km} \mathrm{~s}^{-1} \mathrm{Mpc}^{-1}$ ). Considering the high foreground extinction of our sample, we assume that most of the galaxies classified as late type S/Im were probably misclassified: it is unlikely to find more than a couple of very late-type galaxies at these foreground extinctions. Hence, we have calculated the H I deficiency parameter for the Sdm - I galaxy sample using the average H I content of field galaxies of types Scd and Sd.

The derived $\mathrm{H}$ I deficiency parameters are plotted as a function of cluster-centric distance out to $2 R_{\mathrm{A}}$, as illustrated in Fig. 7. The dispersion is quite large and only a very weak dependence is seen. The least squares fit out to a radius of $2 R_{\mathrm{A}}$ has a slope of $-0.07 \pm 0.12$. A comparison with the respective plot for the Coma cluster (Bravo-Alfaro et al. 2000, their Fig. 4) which is similar to the Norma cluster in its cluster specific properties (Kraan-Korteweg et al. 1996; Woudt 1998), shows that we have only one detection within $0.4 R_{\mathrm{A}}$, whereas Coma shows obvious deficiencies for seven central galaxies. This one detection (WKK 6100) is of unknown morphological type. Revisiting the $R$ - and $J$-band images and taking account of the effect of extinction, it is likely that this galaxy is actually an Sc galaxy. that would make this galaxy more deficient.

However, the fact that we have six non-detections within $d=0.49 R_{\mathrm{A}}$ of the Norma cluster indirectly suggests that the cluster is even more $\mathrm{H}$ I deficient than suggested by Fig. 7. These galaxies must lie below our sensitivity limit. To test this, we looked at the non-detections that have optically determined velocities. Based on that, we can test whether they are part of the cluster or cluster environment. These are identified with open circles in Fig. 5. Nearly all non-detections with optically known velocities have velocities consistent with being part of the cluster (blue open circles with crosses). For these we have calculated upper limits of the H I-mass based on our measured rms, assuming that we would have detected the galaxy if it had an $S / N=3$, and a $50 \%$ line width typical of a spiral galaxy of $200 \mathrm{~km} \mathrm{~s}^{-1}$. These values are added to the sample's H I deficiency parameters, and shown in Fig. 8.

This is not all the data available to test the H I deficiency hypothesis. Vollmer et al. (2001) obtained ATCA radio synthesis imaging observations of the centre of the Norma cluster as well as for two fields just slightly offset from the centre. In all three fields (with a $H P B W$ of $30^{\prime}$ ) they detected only two galaxies (both were not observed by us). That alone presents an indication of H I deficiency. Only one of their two detected galaxies is clearly H I deficient: WKK 6489 lies at $0.36 R_{\mathrm{A}}$ and has an H I deficiency of 1.0, while the other galaxy detected by them, WKK 6801, at $0.75 R_{\mathrm{A}}$ has a normal value of -0.15 .

The ATCA fields contain, however, many more spiral galaxy candidates (the reason why these fields were chosen for observations). We assume that all the spiral galaxies in the ATCA fields actually are members of the Norma cluster. An inspection of the velocity histograms of all known redshifts of galaxies in the Norma cluster (available from Woudt et al. 2008) in rings out to $2 R_{\mathrm{A}}$ confirms that nearly all galaxies within $0.5 R_{\mathrm{A}}$ are members of the cluster, with just a very low number of outliers (see Fig. 9). Even for the outermost ATCA field (at about $0.65 R_{\mathrm{A}}$

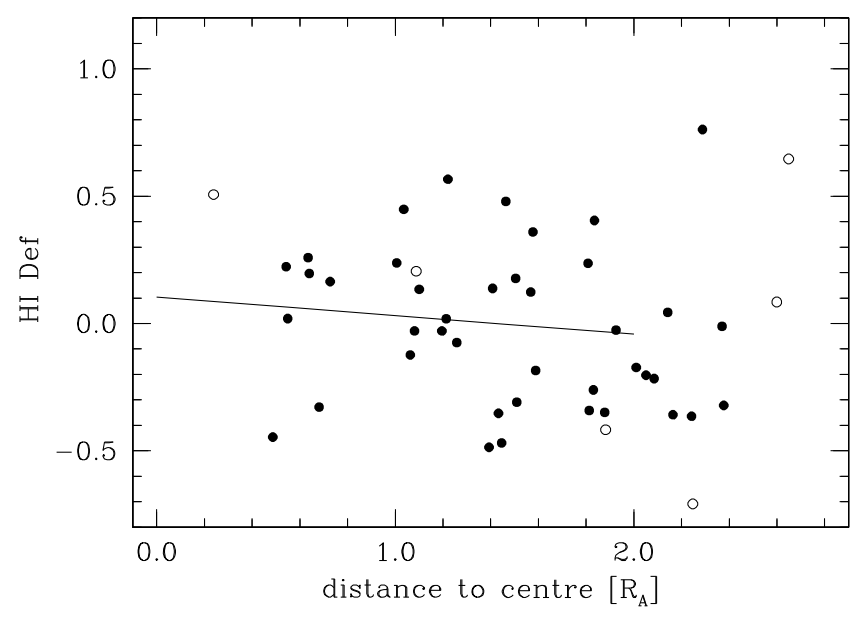

Fig. 7. H I deficiency parameters of Norma cluster are plotted versus distance from the centre of the cluster in Abell radii. Open circles indicate unknown spiral types or early morphological types which are not included in the fitted line. (One $R_{\mathrm{A}}$ corresponds to 1:75).

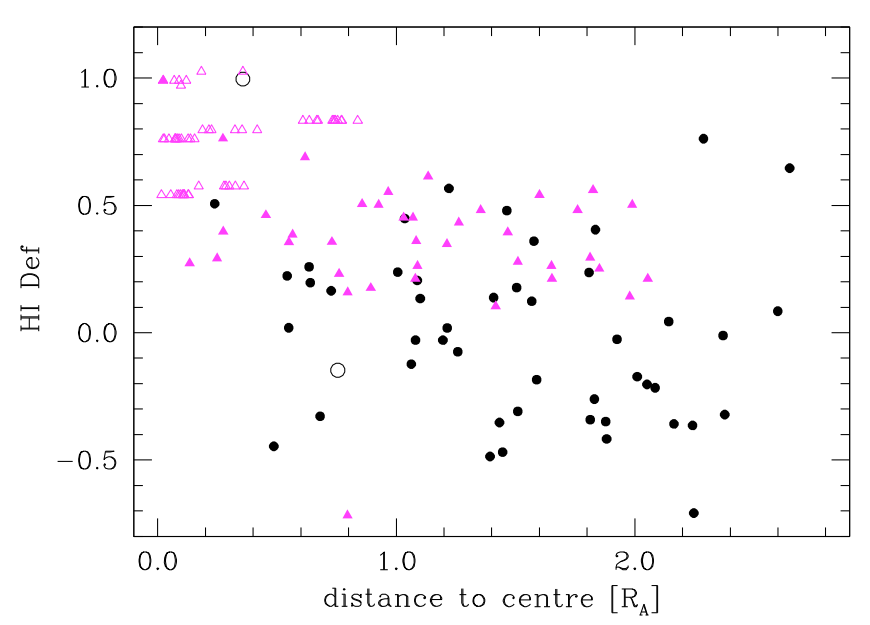

Fig. 8. Same as Fig. 7 but with lower limits for non-detections (triangles). Open symbols stand for ATCA data, filled symbols are from the present paper.

from the cluster centre) contamination by background or foreground galaxies will be minimal.

The ATCA non-detections have a $3 \sigma$ detection limit of about $3 \mathrm{mJy} /$ beam in each velocity channel. Assuming emission to be unresolved with the $30^{\prime \prime}$ beam in each channel, the $3 \sigma$ upper limit on H I mass for these non-detections is $6 \times 10^{8} M_{\odot}$ (following Vollmer et al. 2001, but with $D=67 \mathrm{Mpc}$ rather than $D=79 \mathrm{Mpc}$, and an assumed linewidth of $200 \mathrm{~km} \mathrm{~s}^{-1}$ rather than $150 \mathrm{~km} \mathrm{~s}^{-1}$ ). Taking this limit, the calculated H I deficiency for spiral galaxies in the ATCA fields are also added to Fig. 8 (filled triangles). The addition of these lower limits now reveal a very clear and strong trend of $\mathrm{H}$ I deficiency for galaxies within $0.4 R_{\mathrm{A}}$. For the annulus from $0.4 R_{\mathrm{A}}$ to $1 R_{\mathrm{A}}$ and beyond, the H I deficiency scatters between normal values to considerably deficient galaxies. This is consistent with what has been found for other rich clusters, where clear H I deficiency manifests itself unambiguously only in the innermost core of the cluster $\left(R \lesssim 0.5 R_{\mathrm{A}}\right.$; see e.g., Haynes et al. 1984). 

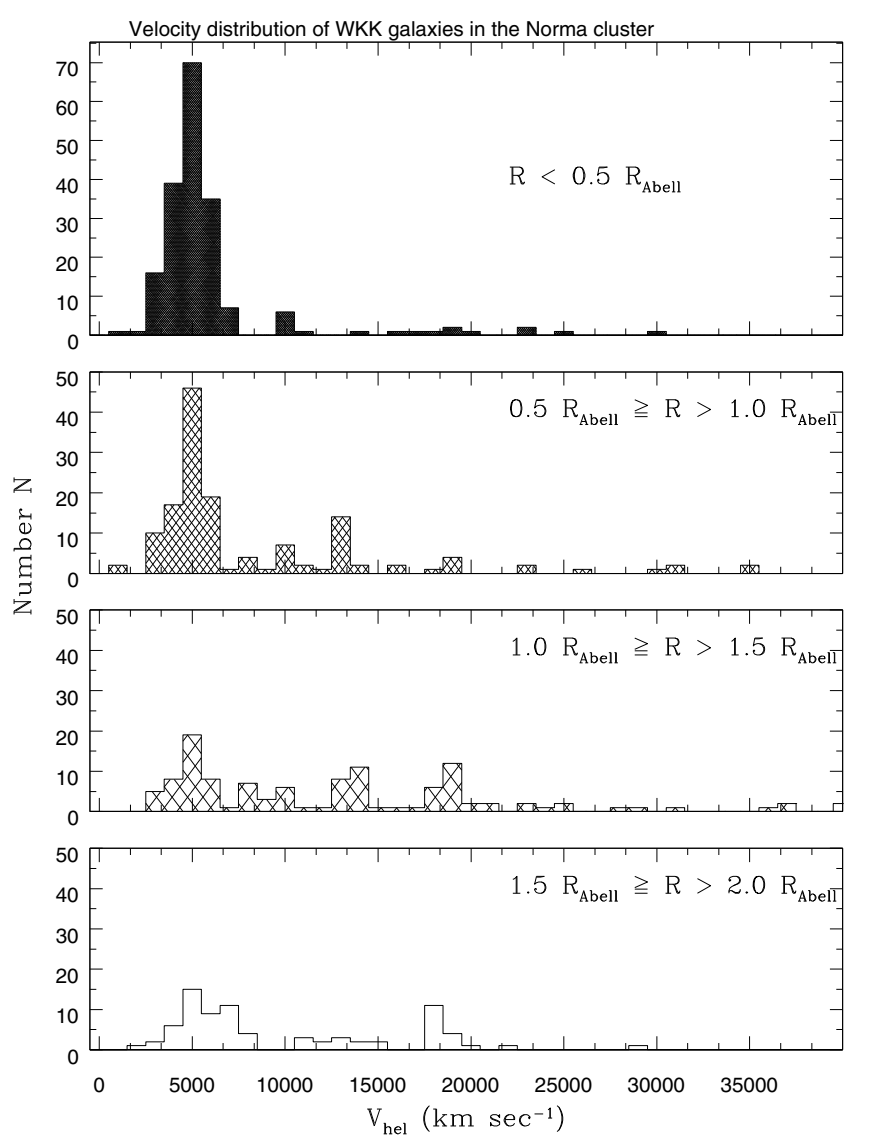

Fig. 9. Velocity distribution of WKK (optical) detections in the Norma region, separated by Abell radii, as indicated.

The non-detection rate can also be used to calculate an HI deficiency fraction as defined by Giovanelli \& Haynes 1985. In our sample, we have one detection with an H I deficiency $>0.3$ within $1 R_{\mathrm{A}}$ as well as 13 lower limits close to or above 0.3. There are an additional 8 detections and 5 nondetections which may have $\mathrm{H}$ I deficiencies just under 0.3 . The H I deficiency fraction in this case is at least 0.58. Böhringer et al. (1996) give an X-ray luminosity for the Norma cluster of $2.2( \pm 0.3) \times 10^{44} \mathrm{erg} \mathrm{s}^{-1}$ in the ROSAT energy band $(0.1-2.4 \mathrm{keV})$. Figure 9 in Giovanelli \& Haynes shows a relationship between the $\mathrm{H}$ I deficiency fraction and X-ray luminosity in the $(0.5-3.0 \mathrm{keV})$ band. The values for the Norma cluster fit well onto that relationship.

For completeness, we have checked for HI deficiency in our other galaxies. There are only two outliers, WKK 1294 with $(l, b, v)=\left(301.7,10^{\circ} 1,1919 \mathrm{~km} \mathrm{~s}^{-1}\right)$ and WKK 1510 with $(l, b, v)=\left(303^{\circ} .4,7^{\circ} 0,2668 \mathrm{~km} \mathrm{~s}^{-1}\right)$. Both are located in the vicinity of the two clusters in Crux area, the CIZA clusters (Clusters in the ZOA, Ebeling et al. 2002) CIZA 1324.7-5736 at $(l, b, v)=\left(307^{\circ}, 5^{\circ}, 5700 \mathrm{~km} \mathrm{~s}^{-1}\right)$ and less rich Cen-Crux clusters $(l, b, v)=\left(306^{\circ}, 5.5,6200 \mathrm{~km} \mathrm{~s}^{-1}\right)$, but not close enough to expect any $\mathrm{H}$ I stripping. It is therefore more likely that the morphological types (S3 and S4, respectively) are wrong, and that these galaxies are of later type. This is supported for WKK 1294, which is bright in the $B$-band but barely visible on the 2MASS $J$-band image. A later type would reduce the HI deficiency value.

\section{Large-scale structure in the Crux and GA regions}

In this section, we will discuss the new HI detections in the context of the known large-scale structures (LSS) in and across the ZOA. The new data is presented together with previously known redshifts in and adjacent to the survey area (extracted from LEDA) in a series of sky projections and redshift cones (Figs. 10 and 11). Care should be taken in the interpretation of these plots as they are based on an "uncontrolled" redshift sample of galaxies.

\subsection{Sky projections}

Figure 10 displays the galaxy distribution in Galactic coordinates in and around the survey region sliced in redshift intervals of width $\Delta v=2000 \mathrm{~km} \mathrm{~s}^{-1}$ out to $8000 \mathrm{~km} \mathrm{~s}^{-1}$ (the first slice runs from $300-2000 \mathrm{~km} \mathrm{~s}^{-1}$ ). Higher velocity slices (8000-12000) are not shown, as the detections for $v>8000 \mathrm{~km} \mathrm{~s}^{-1}$ (see Fig. 4) are too scarce to add useful insight to the LSS at those redshifts.

What is immediately conspicuous in all four panels is that the $\mathrm{H}$ I detections constitute a large fraction of the galaxies with redshifts within the band limited by $A_{B}<3$. 0 and $b<10^{\circ}$ (apart from the H I-deficient galaxies in the Norma cluster). This emphasises that H I-observations, even when dependent on optical (or NIR) pre-identification, are tremendously powerful in mapping LSS of highly obscured galaxies in and across the ZOA. Note that optical redshifts for these survey regions have been obtained earlier with the SAAO 1.9 m telescope (Fairall et al. 1998; Woudt et al. 1999), as well as with multi-object spectroscopy using the $3.6 \mathrm{~m}$ telescope of ESO in Chile (Woudt et al. 2004).

The first slice is quite sparsely populated. The Supergalactic Plane (SGP; de Vaucouleurs 1953) is the most prominent feature, visible here from about $(\ell, b)=\left(335^{\circ},-30^{\circ}\right)$ to $\left(315^{\circ}\right.$, $+30^{\circ}$ ), crossing the Galactic Plane (GP) at $\ell=325^{\circ}$. Most of the detections in this panel lie along the SGP.

In the second panel (2000-4000) the Centaurus Wall (CenW) is the dominant feature (Fairall 1998; Fairall et al. 1998). It enters the panel at about $\left(345^{\circ},-30^{\circ}\right)$ and extends across the ZOA to the Centaurus clusters $\left(302^{\circ},+22^{\circ}\right)$; it is less inclined than the SGP. Apart from a few detections below the GP in Crux, which do not seem to highlight any particular structure, most of the H I-detected galaxies in this panel follow CenW. However, the detections above the GP spread out over a much wider area than would be expected from the quite narrow CenW, i.e., it seems to veer off toward the right $\left(\sim 310^{\circ},+5^{\circ}\right)$, away from CenW. These galaxies probably are the low velocity members of the two CenCrux clusters discussed below which form part of the Norma Great Wall.

Despite the many H I non-detections at the core of the cluster, the Norma cluster and the Norma Great Wall - dubbed so for the first time by Woudt et al. in 1999 - are the prominent structures in the third panel. There are still a few galaxies visible that form part of CenW (blue dots above the GP around the Crux/GA border). But the majority of the detections (mostly red dots) follow the Norma Wall (Woudt et al. 1999). The Norma Wall can be traced from the Pavo II cluster $\left(\sim 332^{\circ},-23^{\circ}\right)$ to the Norma cluster $\left(\sim 325^{\circ},-7^{\circ}\right)$, see also Fig. 11 . It crosses the Galactic Plane at $\sim 320^{\circ}$, and continues with a much shallower slope with respect to the Galactic Plane towards two neighbouring galaxy clusters at slightly higher redshift, the Cen-Crux cluster at $(\ell, b, v) \sim\left(305^{\circ} .4,5^{\circ}, 6200 \mathrm{~km} \mathrm{~s}^{-1}\right)$ (Woudt et al. 1999; Woudt \& Kraan-Korteweg 2001) and the X-ray cluster CIZA J1324.7-736 at $(\ell, b, v) \sim\left(307^{\circ} .4,5^{\circ}, 5700 \mathrm{~km} \mathrm{~s}^{-1}\right)$ (Ebeling et al. 2002). From there, the Wall connects with the Vela cluster (Abell S0639; 

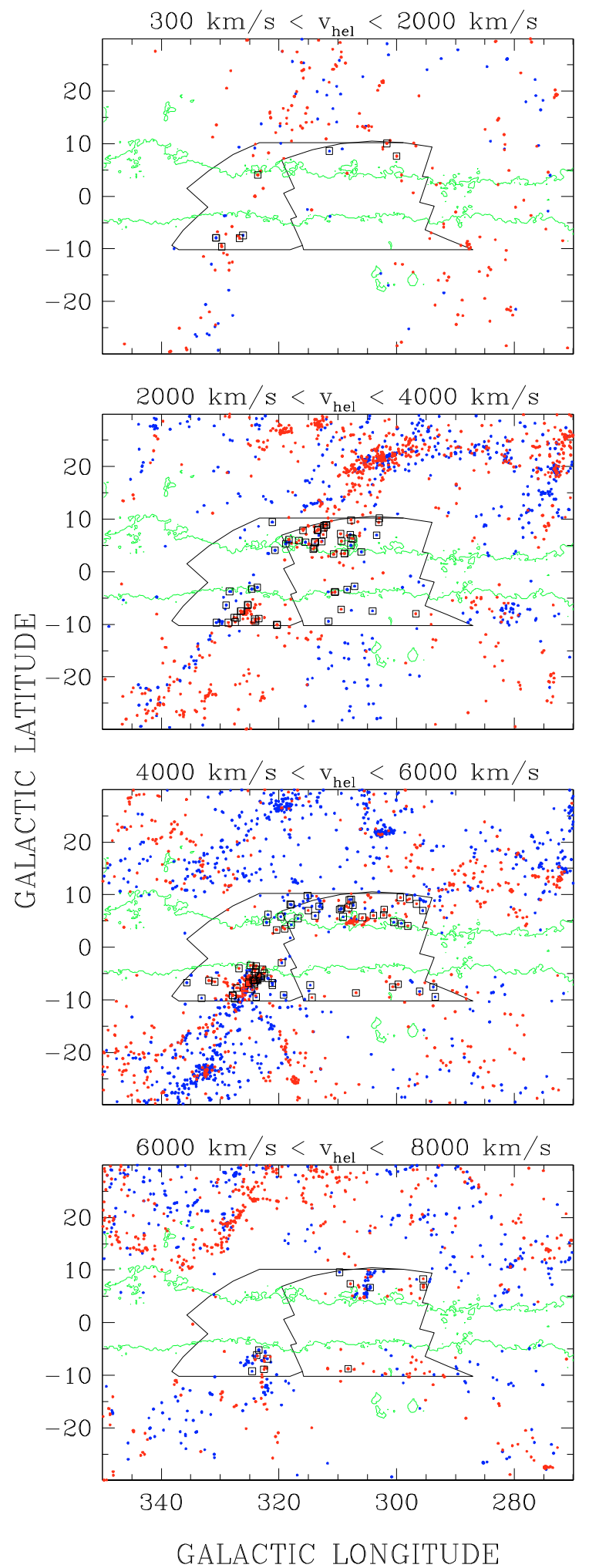

Fig. 10. Sky projections in Galactic coordinates in and around the Crux/GA survey region in redshift intervals of $\Delta v=2000 \mathrm{~km} \mathrm{~s}^{-1}$ for $300<v<8000 \mathrm{~km} \mathrm{~s}^{-1}$. In each panel, the "nearer" galaxies $\left(\Delta v=1000 \mathrm{~km} \mathrm{~s}^{-1}\right)$ have blue symbols, and the more distant red symbols. The squares around the dots identify the new H I detections. The survey area and extinction contour of $A_{B}=3$. 0 are outlined.

$(\ell, b, v)=\left(280^{\circ} .5,-10^{\circ} 9,6326 \mathrm{~km} \mathrm{~s}^{-1}\right)$; Stein 1996). The extent and shape of this wall was suspected in earlier papers (e.g., Kraan-Korteweg et al. 1994) and strongly supported in more recent work (Woudt et al. 2004; Kraan-Korteweg 2005; Ebeling et al. 2005; Radburn-Smith et al. 2006). A sketch of this Wall, as well as the Centaurus filament, is given with Fig. 4 in Radburn-Smith et al. (2006).

In the final panel, most of the HI detections are found at the position of the Norma cluster and the two Crux clusters. They most certainly are high velocity dispersion outliers of the massive clusters (finger-of-God effect) prominent in the previous panel, rather than an indication of galaxy agglomerations at this higher velocity range.

In conclusion, the new $\mathrm{H}$ I detections mostly help to delineate filaments and walls deeper into the ZOA. They provide supporting evidence that the Norma Wall crosses the ZOA, but then turns away from CenW towards Vela. A much smaller number of galaxies seem to form part of the general field. The slices also indicate that the $\mathrm{H}$ I observations of galaxies allow the mapping of LSS to quite high dust column-densities, higher than can be achieved with optical spectroscopy.

\subsection{Pie diagrams}

The above findings are confirmed with Fig. 11, which shows the new detections in a composite of pie diagrams that cover a wider area than the previous figure to reveal the overall LSS in this part of the sky. The two top panels show redshift slices out to $12000 \mathrm{~km} \mathrm{~s}^{-1}$, the maximum redshift range of our HI observations. They are $30^{\circ}$ wide in longitude, with the left one centred on the GA survey region $\left(340^{\circ}>\ell>310^{\circ}\right)$ and the right one centred on the Crux survey region $\left(310^{\circ}>\ell>280^{\circ}\right)$. Note that there is a longitude overlap of $10^{\circ}$ to facilitate the visualisation of the structures running from one wedge diagram to the next. The pie diagram for these two longitude strips run from $-45^{\circ}$ across the $\mathrm{ZOA}$ to $+45^{\circ}$. The bottom panel displays a pie diagram of the $\mathrm{ZOA}\left(|b| \leq 10^{\circ}\right)$ for the longitude range $360^{\circ}-270^{\circ}$ as in the sky projection plot in the middle panel. The middle panel displays the projected LSS distribution of galaxies out to $12000 \mathrm{~km} \mathrm{~s}^{-1}$, with blue dots marking the distance range of Norma and the Norma Wall (3000-6500 $\mathrm{km} \mathrm{s}^{-1}$ ), magenta the nearer galaxies $\left(300-3000 \mathrm{~km} \mathrm{~s}^{-1}\right)$, and cyan the distant galaxies $\left(6500-12000 \mathrm{~km} \mathrm{~s}^{-1}\right)$. This panel is meant for orientation and interpretation of the pie diagrams. The plot is collapsed in latitude.

The pie diagrams confirm that H I-observations of optically selected galaxies are quite successful in reducing the ZOA down to $|b|=5^{\circ}$, at least out to redshifts of about $6000 \mathrm{~km} \mathrm{~s}^{-1}$ (see also Fig. 4). For higher velocities the detection efficiency drops substantially and the ZOA remains increasingly empty. Such a drop in the detection rate at similar velocities can also seen in the systematic, blind deep Parkes H I ZOA survey (HIZOA; $|b| \leq 5^{\circ}$; see, e.g., Fig. 13 in Kraan-Korteweg 2005). This is not surprising given that the rms and velocity range of both surveys are similar. In that sense, the pointed Parkes H I observations presented here, which are quite successful in the range $5^{\circ} \lessgtr|b| \lesssim 10^{\circ}$, are in fact complementary to the deep Parkes HIZOA survey of the inner optically opaque ZOA (Henning et al., in prep.; for preliminary results see Henning et al. 2005; Kraan-Korteweg et al. 2005).

The most prominent feature in the GA cone diagram (top left panel) is the Norma cluster with its finger-of-God ranging between $2500-6500 \mathrm{~km} \mathrm{~s}^{-1}$ evident at $\left(b=-7^{\circ}\right)$. The cluster stands out even more prominently in the bottom panel which displays the ZOA slice. The H I detections (red dots) mostly hover around Norma. The cluster itself seems embedded in a walllike structure centred at a mean velocity of $4500-5000 \mathrm{~km} \mathrm{~s}^{-1}$ which can be traced from about $b \sim-30^{\circ}$ to the Norma cluster 

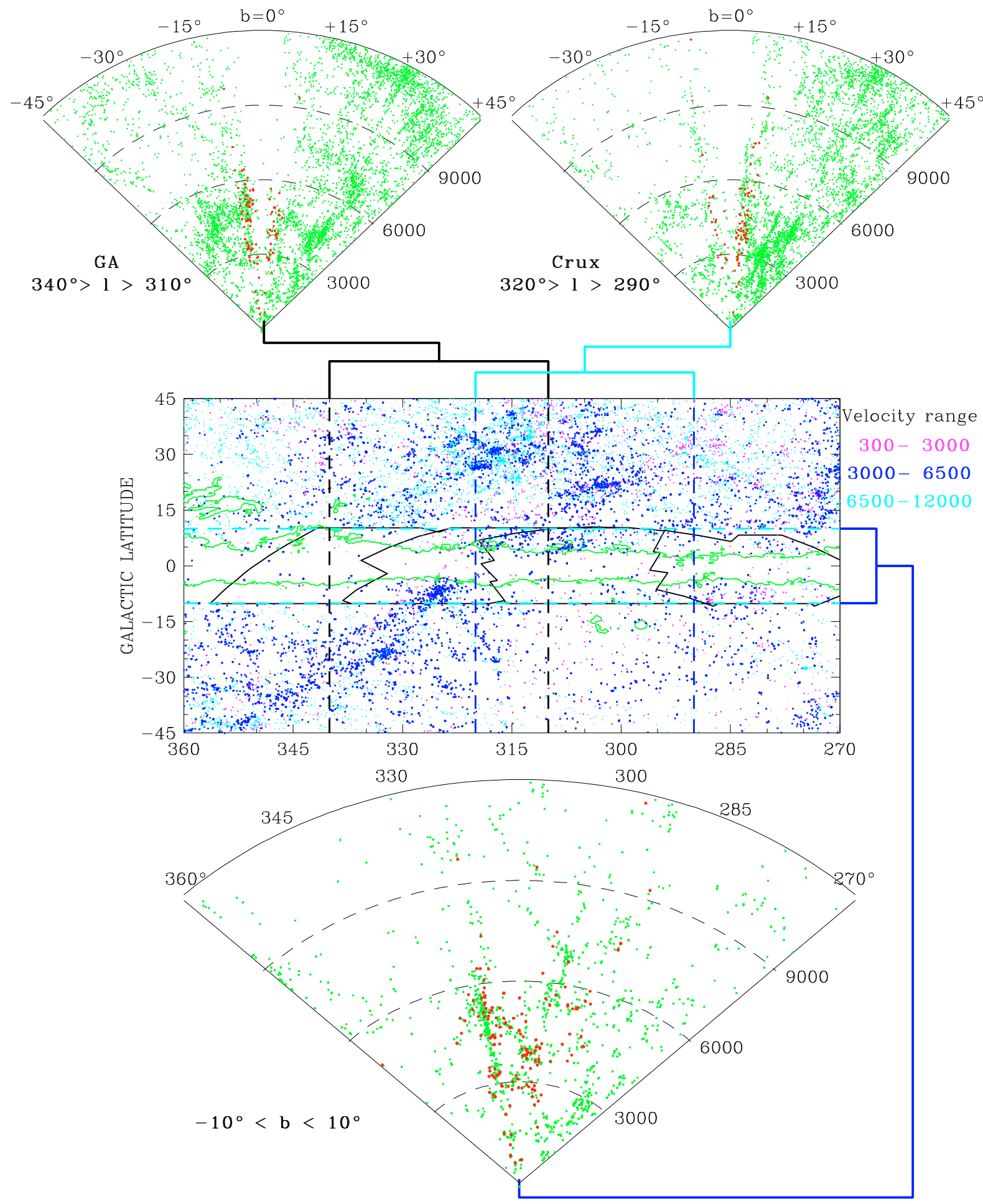

Fig. 11. Large scale structures in and around the Crux/GA region. The top two redshift cones are centred on the GA and Crux regions, respectively, and cover the latitude range $b \leq \pm 45^{\circ}$ out to $v=12000 \mathrm{~km} \mathrm{~s}^{-1}$. The bottom panel displays a redshift cone of the ZOA $\left(|b| \leq 10^{\circ}\right)$. Red and green dots represent the Parkes H I detections and previously determined redshifts from LEDA, respectively. The middle panel shows the sky distribution of galaxies out to the same redshift. It is meant as a guide in the interpretation of the redshift cones.

$\left(b=-7^{\circ}\right)$ where we lose it in the ZOA. This is also evident in the middle panel where we see part of the wall between the Pavo II cluster (Abell S0805 at $332^{\circ},-24^{\circ}, 4200 \mathrm{~km} \mathrm{~s}^{-1}$ ) and Norma. A weak continuation of the wall is visible on the other side of the $\mathrm{GP}$, together with an overdensity at slightly lower velocities. The latter is probably related to CenW (see also panel 2 in Fig. 10).
We find a clearer continuation of these structures in the Crux cone (top right panel). Most of the detections lie above the GP. Hardly any detections or possible extension of the Norma cluster/wall exists below the GP. The galaxies either form part of CenW which connects with the Centaurus clusters visible in this cone at lower velocities at $b \simeq 20-30^{\circ}$, or with the higher 
velocity Crux clusters (Cen-Crux and CIZA J1324.7-736). The latter two also stand out in the ZOA-longitude slice displayed in the bottom panel. The link with the galaxy cluster in Vela (Abell S0639) is not visible here as the cluster with $(\ell, b, v)=$ $\left(280^{\circ} .5,-10.9,6326 \mathrm{~km} \mathrm{~s}^{-1}\right)$ (Stein 1996) lies just beyond the Crux slice (and just beyond the latitude limit of the ZOA slice).

The bottom panel shows the galaxy distribution in the traditional "optical" ZOA, i.e., for $|b| \lesssim 10^{\circ}$ for a major fraction of the southern ZOA, where near-infrared surveys are also highly incomplete (e.g., Fig. 9 in Kraan-Korteweg 2005). It is satisfying to see that real LSS including voids are now emerging within the ZOA. The H I data follow again the previously identified structures, which on average lie at slightly higher latitudes. Distinct features are the Norma cluster with its enormous velocity dispersion, as well as the Cen-Crux and CIZA cluster, clearly identifiable as clusters. As a clump (rather than a finger of God) we find an overdensity in red and green dots at about $4000 \mathrm{~km} \mathrm{~s}^{-1}$ that most certainly is a signature of the crossing of the Centaurus Wall across the ZOA.

The wall-like structure of Norma is not obvious in this ZOA pie diagram, as the larger structures - from Pavo to Norma and from the Crux clusters to Vela - lie just beyond the ZOA, whereas the inner ZOA $\left(|b|<5^{\circ}\right)$ remains unprobed by this project due to the optical pre-selection of spiral galaxy candidates. However, the pie diagram will soon be filled by the deep Parkes HIZOA survey, where indeed evidence is found for the continuation of the Centaurus Wall and the Norma (or GA) Wall suggested here (see Kraan-Korteweg 2005; Henning et al. 2005, for preliminary results).

\section{Summary}

This paper presents pointed $21 \mathrm{~cm}$ spectral line (H I) observations obtained with the $64 \mathrm{~m}$ Parkes radio telescope of partially to heavily-obscured spiral galaxies uncovered in the deep optical search for galaxies in the Crux and GA regions of the ZOA (Woudt \& Kraan-Korteweg 2001). Out of a total of 314 observed galaxies we have detected 162 . Due to the high density of galaxies in some areas (in particular near and in the Norma cluster), a number of pointings contain more than one detection. On the other hand, some pointings of a non-detection show a chance detection of a different galaxy (with a distance to the beam centre of up to $20^{\prime}$ ).

The average rms for the detections is $4.2 \mathrm{mJy} ; 85 \%$ of all detections have an rms within the range 2-6 mJy. Non-detections have a slightly higher rms of $4.7 \mathrm{mJy}$. The mean peak flux density is $46 \mathrm{mJy}$ (with a median of $39 \mathrm{mJy}$ ), which is significantly lower than the average detection limit of the HIPASS detections $(\sim 70 \mathrm{mJy})$ but similar to HIZOA (Henning et al. 2005; Kraan-Korteweg et al. 2005). The H I parameters compare well with those found with HIPASS, and the velocities compare well with optical velocities found in the literature.

The detection rate in the survey area is $52 \%$, slightly higher than in the Hydra/Antlia survey region (45\%) obtained in the same way (Paper I). The exception is the core of the Norma cluster: within $1 R_{\mathrm{A}}$, the detection rate drops to $41 \%$ as expected in case of H I deficiency. To explore this further, we have derived $\mathrm{HI}$ deficiency parameters for all galaxies in and around the Norma cluster. Although only a few galaxies have actually been detected within $1 R_{\mathrm{A}}$, the lower limits of non-detections confirm that the galaxies in the Norma cluster are on average strongly $\mathrm{H}$ I deficient within $0.4 R_{\mathrm{A}}$. Including three fields observed with ATCA in the Norma cluster at an earlier stage
(Vollmer et al. 2001) and calculating the upper limits for nondetected spiral galaxies strengthens this conclusion. It shows furthermore that non-detected spiral galaxies between $0.4 R_{\mathrm{A}}$ and $1.0 R_{\mathrm{A}}$ also exhibit $\mathrm{H}$ I deficiencies. The observed trend is similar to that seen in the Coma cluster within $0.4 R_{\mathrm{A}}$ (Bravo-Alfaro et al. 2000) and to other massive, X-ray bright galaxy clusters within $1.0 R_{\mathrm{A}}$ (Giovanelli \& Haynes 1985).

The H I detections delineate large-scale structures, such as filaments, walls and voids, deeper into the ZOA than any of the other previous optical redshift follow-ups of the optically identified galaxies. Most low-velocity detections lie along the SGP, while intermediate-velocity galaxies follow the CenW. The higher-velocity galaxies (5000-6500 $\mathrm{km} \mathrm{s}^{-1}$ ) support evidence that the Norma Wall crosses the ZOA, but then turns from the CenW towards Vela. Only a small number of the detected galaxies seem to form part of the general field.

We have shown that H I observations, even when dependent on optical (or NIR) pre-identification, are quite successful in reducing the (redshift) ZOA down to $|b| \gtrsim 5^{\circ}$ and in mapping LSS of highly obscured galaxies in and across the ZOA, at least out to redshifts of about $6000 \mathrm{~km} \mathrm{~s}^{-1}$. For higher velocities the detection efficiency drops substantially and the ZOA remains increasingly empty. Our observations are complementary to the deep Parkes HIZOA survey of the inner optically opaque ZOA at $|b| \leq 5^{\circ}$ (Kraan-Korteweg et al. 2005; Henning et al. 2005; Henning et al., in prep).

With the future Square Kilometer Array (SKA) pathfinders, the Australian ASKAP and the South African MeerKAT, the situation will be greatly improved: we will be able to close the redshift ZOA to even lower latitudes and to map the LSSs to higher redshifts and lower H I masses. For example, MeerKAT will not only be able to fully map the GA but also the Shapley cluster concentration and thus possibly solve the GA/Shapley controversy on what is the dominant contributor to the dipole motion of the universe (Kraan-Korteweg et al. 2009, and references therein).

Acknowledgements. We first of all would like to thank P. A. Woudt for many valuable discussions and the referee for many valuable suggestions. We have made use of the Lyon-Meudon Extragalactic Database (LEDA), supplied by the LEDA team at the Centre de Recherche Astronomique de Lyon, Observatoire de Lyon, and of the NASA/IPAC Extragalactic Database (NED), which is operated by the Jet Propulsion Laboratory, Caltech, under contract with the National Aeronautics and Space Administration. Furthermore, this research has made use of the Digitized Sky Surveys (produced at the Space Telescope Science Institute under US Government grant NAG W-2166) and of the HIPASS database provided by the ATNF under the auspices of the Multibeam Survey Working Group. RKK thanks the South African National Research Foundation for support.

\section{References}

Arp, H. C., \& Madore, B. F. 1987, A Catalog of Southern Peculiar Galaxies and Associations (Cambridge: Cambridge University Press)

Banks, G. D., Disney, M. J., Knezek, P. M., et al. 1999, ApJ, 524, 612

Böhringer, H., Neumann, D. M., Schindler, S., \& Kraan-Korteweg, R. C. 1996, ApJ, 467, 168

Bravo-Alfaro, H., Cayatte, V., van Gorkom, J. H., \& Balkowski, C. 2000, AJ, 119,580

Buta, R. 1995, ApJS, 96, 39

Coté, S., Freeman, K. C., Carignan, C., \& Quinn, P. J. 1997, AJ, 114, 1313

de Vaucouleurs, G. 1953, AJ, 58, 30

de Vaucouleurs, G., de Vaucouleurs, A., \& Corwin, H. G. 1976, 2nd Reference Catalogue of Bright Galaxies (RC2) (Austin: University of Texas Press)

de Vaucouleurs, G., de Vaucouleurs, A., Corwin, H. G., et al. 1991, Third

Reference Catalogue of Bright Galaxies (RC3) (New York: Springer)

Di Nella, H., Paturel, G., Walsh, A. J., et al. 1996, A\&AS, 118, 311

Di Nella, H., Couch, W. J., Parker, Q. A., \& Paturel, G. 1997, MNRAS, 287, 472 
Djorgovski, S., Thompson, D. J., de Carvalho, R. R., \& Mould, J. R. 1990, AJ, 100,599

Donley, J. L., Staveley-Smith, L., \& Kraan-Korteweg, R. C. 2005, AJ, 129, 220

Dressler, A. 1991, ApJS, 75, 241

Dreyer, J. L. E. 1888, Mem. Mon. R. A. S., 49, 1

Dreyer, J. L. E. 1908, Mem. Mon. R. A. S., 59, 105

Ebeling, H., Mullis, C. R., \& Tully, R. B. 2002, ApJ, 580, 774

Ebeling, H., Kocevski, D., Tully, R. B., \& Mullis, C. R. 2005, in Nearby LargeScale Structures and the Zone of Avoidance, ed. A. P. Fairall, \& P. A. Woudt (San Francisco: ASP), ASP Conf. Ser., 329, 83

Epchtein, N., Batz, B. de, Capoani, L., et al. 1997, Messenger, 87, 27

Fairall, A. P. 1983, MNRAS, 203, 47

Fairall, A. P. 1998, Large-Scale Structures in the Universe (Chichester: John Wiley \& Sons Ltd in association with Praxis Publishing)

Fairall, A. P., \& Woudt, P. A. 2005, Nearby Large-Scale Structures and the Zone of Avoidance (San Francisco: ASP), ASP Conf. Ser., 329

Fairall, A. P., \& Woudt, P. A. 2006, MNRAS, 366, 267

Fairall, A. P., Woudt, P. A., \& Kraan-Korteweg, R. C. 1998, A\&AS, 127, 463

Fisher, K. B., Huchra, J., Davis, M., et al. 1995, ApJS, 100, 69

Giovanelli, R., \& Haynes, M. P. 1985, ApJ, 292, 404

Hasegawa, T., Wakamatsu, K.-I., Malkan, M., et al. 2000, MNRAS, 316, 326

Haynes, M. P., Giovanelli, R., \& Chincarini, R. 1984, ARvA\&A, 22, 445

Henning, P. A., Staveley-Smith, L., Ekers, R. D., et al. 2000, AJ, 119, 2686 (HIZSS)

Henning, P. A., Kraan-Korteweg, R. C., \& Staveley-Smith, L. 2005, in Nearby Large-Scale Structures and the Zone of Avoidance (San Francisco: ASP), ed. A. P. Fairall, \& P. A. Woudt, ASP Conf. Ser., 329, 199

Huchra, J. P., Geller, M. J., Clemens, C. M., Tokarz, S. P., \& Michel, A. 1995, Harvard Smithsonian Center for Astrophysics, The CFA redshift catalogue

Huchtmeier, W. K., Karachentsev, I. D., \& Karachentseva, V. E. 2001, A\&A, 377,801

Juraszek, S. J., Staveley-Smith, L., Kraan-Korteweg, R. C., et al. 2000, AJ, 119, 1627

Lauberts, A. 1982, The ESO/Uppsala Survey of the ESO (B) Atlas, ESO: Garching

Lauberts, A., \& Valentijn, E. A. 1989, The Surface Photometry Catalogue of the ESO-Uppsala Galaxies, ESO: Garching

Karachentsev, I. D., Karachentseva, V. E., \& Parnovsky, S. L. 1993, Flat Galaxy Catalog, ANac, 314, 97

Koribalski, B., \& Dickey, J. M. 2004, MNRAS, 348, 1255

Koribalski, B., Staveley-Smith, L., \& Kilborn, V. A. 2004, AJ, 128, 16 (HIPASS-BGC)

Kraan-Korteweg, R. C. 2000, A\&AS, 141, 123

Kraan-Korteweg, R. C. 2005, in From Cosmological Structures to the Milky Way (New York: Wiley), ed. S. Röser, Rev. Mod. Astron., 18, 48

Kraan-Korteweg, R. C., \& Lahav, O. 2000, A\&ARv, 10, 211

Kraan-Korteweg, R. C., Cayatte, V., Fairall, A. P., et al. 1994, in Unveiling Large-Scale Structures behind the Milky Way, ed. C. Balkowski, \& R. C. Kraan-Korteweg (San Francisco: ASP), ASP Conf. Ser., 67, 99

Kraan-Korteweg, R. C., Fairall, A. P., \& Balkowski, C. 1995, A\&A, 297, 617

Kraan-Korteweg, R. C., Woudt, P. A., Cayatte, V., et al. 1996, Nature, 379, 51

Kraan-Korteweg, R. C., Henning, P. A., \& Andernach, H. 2000, Mapping the Hidden Universe: The Universe behind the Milky Way - The Universe in H I (San Francisco: ASP), ASP Conf. Ser., 218

Kraan-Korteweg, R. C., Henning, P. A., \& Schröder, A. C. 2002, A\&A, 391, 887 (Paper I)

Kraan-Korteweg, R. C., Staveley-Smith, L., Donley, J., Koribalski, B., \& Henning, P. A. 2005, in Maps of the Cosmos, ed. M. Colless, \& L. Staveley-Smith (San Francisco: ASP), IAU Symp., 216, 203

Kraan-Korteweg, R. C., van der Heyden, K. J., Cluver, M. E., \& Woudt, P. A. 2009, in First Middle East-Africa Regional IAU Meeting (MEARIM), ed. A. Hady (Cairo University Press), in press [arXiv: 0810.3929]

Mathewson, D. S., \& Ford, V. L. 1996, ApJS, 107, 97

Multibeam Survey Working Group, HI Parkes All Sky Survey Final Catalog 2006 (HIPASS)

Mould, J. R., Han, M. S., Roth, J., et al. 1991, ApJ, 383, 467

Nilson, P. 1973, Uppsala General Catalog of Galaxies, Uppsala, University of Uppsala

Paturel, G., Theureau, G., Bottinelli, L., et al. 2003, A\&A, 412, 57

Radburn-Smith, D. J., Lucey, J. R., Woudt, P. A., Kraan-Korteweg, R. C., \& Watson, F. G. 2006, MNRAS, 369, 1131

Roman, A. T., Iwata, I., \& Saito, M. 2000, ApJS, 127, 27

Ryan-Weber, E., Koribalski, B. S., Staveley-Smith, L., et al. 2002, AJ, 124, 1954

Saito, M., Ohtani, H., Baba, A., et al. 1991, PASJ, 43, 449

Sanders, D. B., Egami, E., Lipari, S., Mirabel, I. F., \& Soifer, B. T. 1995, AJ, 100,1993

Schlegel, D. J., Finkbeiner, D. P., \& Davis, M. 1998, ApJ, 500, 525

Schneider, S. E., Helou, G., Salpeter, E. E., \& Terzian, Y. 1986, AJ, 92, 472
Schneider, S. E., Thuan, T. X., Magri, C., \& Wadiak, J. E. 1990, ApJS, 72, 245 Skrutskie, M. F., Cutri, R. M., Stiening, R., et al. 2006, AJ, 131, 116 Solanes, J. M., Giovanelli, R., \& Haynes, M. H. 1996, ApJ, 461, 609 Staveley-Smith, L. 1985, Ph.D. Thesis, University of Manchester

Staveley-Smith, L., Juraszek, S., Henning, P. A., Koribalski, B. S., \& Kraan-Korteweg, R. C. 2000, in Mapping the Hidden Universe: The Universe behind the Milky Way - The Universe in HI, ed. R. C. Kraan-Korteweg, P. A. Henning, \& H. Andernach (San Francisco: ASP), ASP Conf. Ser., 218, 207 Stein, P. 1996, A\&AS, 116, 203

Strauss, M. A., Huchra, J. P., Davis, M., et al. 1992, ApJS, 82, 29

Theureau, G., Hanski, M. O., Coudreau, N., Hallet, N., \& Martin, J.-M. 2007, A\&A, 465, 71

Two Micron All Sky Survey team 2003, 2MASS extended objects, Final release Vauglin, I., Rousseau, J., Paturel, G., et al. 2002, A\&A, 387, 1

Visvanathan, N., \& van den Bergh, S. 1992, AJ, 103, 1057

Visvanathan, N., \& Yamada, T. 1996, ApJS, 107, 521

Vollmer, B., Cayatte, V., van Driel, W., et al. 2001, A\&A, 369, 432

Woudt, P. A. 1998, Ph.D. Thesis, University of Cape Town

Woudt, P. A., \& Kraan-Korteweg, R. C. 2001, A\&A, 380, 441

Woudt, P. A., Kraan-Korteweg, R. C., \& Fairall, A. P. 1999, A\&A, 352, 39

Woudt, P. A., Kraan-Korteweg, R. C., Cayatte, V., Balkowski, C., \& Felenbok, P. 2004, A\&A, 415, 9

Woudt, P. A., Kraan-Korteweg, R. C., Lucey, J., Fairall, A. P., \& Moore, S. A. W. 2008, MNRAS, 383, 445

Yahil, A., Tammann, G. A., \& Sandage, A. 1977, ApJ, 217, 903

Yamada, T., Takata, T., Djamaluddin, T., et al. 1993, ApJS, 89, 57

\section{Appendix A: Notes to individual galaxies}

In the following we discuss cases where the cross-identification is not straightforward or where the signal is a combination of the signals from two or more galaxies. In many cases we quote data from Tables 1-4 (including the optical galaxy catalogue by Woudt \& Kraan-Korteweg 2001), notably morphological types, sizes, and optical velocities. We have also made use of DSS ${ }^{4}$ images and of images from the DENIS (Epchtein et al. 1997) and 2MASS (Skrutskie et al. 2006) surveys.

WKK 0491/WKK 0512: there is a second detection in the beam of WKK 0491. The small lopsided signal is probably due to WKK $0512\left(d=12 ! 4,28^{\prime \prime} \times 8^{\prime \prime}\right.$, SL) which is the largest latetype spiral in the vicinity. WKK 0493 at $d=10$ '2 $\left(28^{\prime \prime} \times 6^{\prime \prime}, \mathrm{L}\right)$ is an early type galaxy and less likely to be the candidate.

WKK 0969/WKK 1117: there is a detection in the OFF observation of WKK 1117, close to but separated from its signal. The nearest candidate is WKK 0969 at $d=8.5\left(16^{\prime \prime} \times 12^{\prime \prime}\right.$, unknown type, $A_{B}=33^{\mathrm{m}} 4$ ), which is also the only galaxy found by 2MASS in this area.

WKK 1696 has also been detected in the beam of WKK 1694 (not detected) at $d=8.7$ with $v=6680 \mathrm{~km} \mathrm{~s}^{-1}$, $\Delta V_{50}=300 \mathrm{~km} \mathrm{~s}^{-1}, \Delta V_{20}=318 \mathrm{~km} \mathrm{~s}^{-1}, I=2.47 \mathrm{Jy} \mathrm{km} \mathrm{s}^{-1}$, $\mathrm{rms}=2.4 \mathrm{mJy}$.

WKK 2163: the ID is ambiguous. The observed velocity is $v=3533 \mathrm{~km} \mathrm{~s}^{-1}$, and WKK 2160 at $d=3 ! 3\left(27^{\prime \prime} \times 24^{\prime \prime}, \mathrm{S}\right)$ has an optical velocity of $3512 \pm 58 \mathrm{~km} \mathrm{~s}^{-1}$ (FW98). It is possible that we have observed WKK 2160, but if the two galaxies are genuine companions the signal probably comes from WKK 2163 $\left(74^{\prime \prime} \times 56^{\prime \prime}\right.$, S6) which is the larger of the two. HIPASS can barely resolve the positions, but seems to favour WKK 2163.

WKK 2245: it is possible that WKK 2240 (ESO173-G015, $\left.d=2 ! 6, \mathrm{~S}, 85^{\prime \prime} \times 12^{\prime \prime}\right)$ with an optical velocity of $v=3006 \pm$ $36 \mathrm{~km} \mathrm{~s}^{-1}$ (SH92) contributes to the signal.

WKK 2372/WKK 2402: the distance between the galaxies is $d=16$ ' 1 , both have been detected at similar velocities, but the profiles are not confused.

\footnotetext{
${ }^{4}$ The STScI Digitized Sky Survey.
} 
WKK 2377/WKK 2388/WKK 2406: this is a group of four galaxies: WKK 2377 (S7:, 70" $\left.\times 23^{\prime \prime}\right)$, WKK $2375($ S5, 62" $\times$ $56^{\prime \prime}, 4290 \pm 37 \mathrm{~km} \mathrm{~s}^{-1}$ FH95), WKK $2388\left(\mathrm{~S} 5,36^{\prime \prime} \times 16^{\prime \prime}\right.$, $\left.3976 \pm 40 \mathrm{~km} \mathrm{~s}^{-1}, \mathrm{FH} 95\right)$, and WKK $2406\left(\right.$ SL, 55" $\left.\times 38^{\prime \prime}\right)$. WKK 2388 lies between WKK 2377 and WKK 2406 and the pointing of WKK 2388 shows a confusion of the two latter profiles at $v \simeq 4100-4400 \mathrm{~km} \mathrm{~s}^{-1}$. The signal at $v \simeq$ $3800-4050 \mathrm{~km} \mathrm{~s}^{-1}$ belongs to WKK 2388 proper and the line width is assumed to be unaffected. However, due to difficulties with fitting a good baseline the flux density is somewhat uncertain. Using HIPASS we have found that WKK 2375 does not add (significantly) to the combined signal. The observations of WKK 2377 and WKK 2406 at their respective pointings are not confused at the noise level.

WKK 2390/WKK 2392: the close galaxy pair (at $d=1.2$ and $d=3 ! 2$, respectively, from the pointing position) is unresolved, and the parameters given in Table 1 refer to the full profile. The quoted velocity by VY96 refers to the IRAS detection which is also unresolved.

WKK 2503: also observed with HIZSS, the spectrum shows a profile reminiscent of the blended signal of two objects. WKK 2503 has a bright bulge and large faint halo with a bright star superimposed close to the bulge. No other galaxy in this area is visible either in the optical or in the NIR. At an extinction of $A_{B}=3.7$ a late-type/LSB galaxy, WKK 2503B, could be invisible even in the NIR. Table 1 gives the parameters of the full profile. The two velocities found in the literature refer to H I observations and are equally unresolved.

WKK 2576/WKK 2595/WKK 2597: the observed profile is a combination of the signals from three galaxies: WKK 2595 $\left(\right.$ S6, 102" $\left.\times 40^{\prime \prime}\right)$ and WKK 2597 (S5, 59" $\times 47^{\prime \prime}, 3973 \pm$ $46 \mathrm{~km} \mathrm{~s}^{-1}$, SH92) are a close galaxy pair $(d=1.2)$; according to HIPASS, WKK 2576 at $d=8.4\left(\mathrm{~S} 5,86^{\prime \prime} \times 75^{\prime \prime}\right.$, $3948 \pm 70 \mathrm{~km} \mathrm{~s}^{-1}$, DN97) has a strong narrow Hi profile. Table 1 gives the measurement of the full profile for the unresolved pair WKK 2595/WKK 2597, while, through comparison with HIPASS, the width and velocity of the peak at $v \simeq$ $3800-3950 \mathrm{~km} \mathrm{~s}^{-1}$ is given for WKK 2576 . By removing this peak we have re-measured the width and velocity of the underlying low-intensity profile for the close galaxy pair and found $v=3889 \mathrm{~km} \mathrm{~s}^{-1}, \Delta V_{50}=266 \mathrm{~km} \mathrm{~s}^{-1}, \Delta V_{20}=313 \mathrm{~km} \mathrm{~s}^{-1}$.

WKK 2640/WKK 2644: there are two detections in the beam of WKK 2640: the narrow spike at $v=3705 \mathrm{~km} \mathrm{~s}^{-1}$ is WKK $2640\left(\mathrm{I}, 51^{\prime \prime} \times 42^{\prime \prime}\right)$, while the detection at $v=$ $9404 \mathrm{~km} \mathrm{~s}^{-1}$ is WKK $2644\left(\mathrm{SM}, 26^{\prime \prime} \times 9^{\prime \prime}\right)$ with an optical velocity of $9406 \pm 100 \mathrm{~km} \mathrm{~s}^{-1}$ (WK04) at $d=4 ! 3$. Due to the very lopsided profile of WKK 2644 the high-velocity end is uncertain.

WKK 2844/WKK 2863: two galaxies contribute to the detected signal. HIPASS shows that WKK $2863\left(\right.$ S5, $98^{\prime \prime} \times 83^{\prime \prime}$, $3778 \pm 30 \mathrm{~km} \mathrm{~s}^{-1}$, SE95) at $d=8$.7 has a strong profile with $v \simeq 3600-3850 \mathrm{~km} \mathrm{~s}^{-1}$. At the position of WKK 2844 the observed profile is smaller but extends to $v \simeq 3950 \mathrm{~km} \mathrm{~s}^{-1}$; it is therefore assumed that WKK 2844 has been detected but it remains unresolved, with a velocity slightly larger than the one for WKK 2863. Table 1 gives the parameters of the full profile for WKK 2863, which is considered to be the main contributor to the signal.

WKK 2924/WKK 2938: there are two detections in the beam of WKK 2924: WKK $2938\left(\mathrm{~L}, 34^{\prime \prime} \times 22^{\prime \prime}\right)$ at $d=7 ! 3$ has an optical velocity of $3024 \pm 157 \mathrm{~km} \mathrm{~s}^{-1}$ (FW98) which agrees with the narrow peak at $v=2864 \mathrm{~km} \mathrm{~s}^{-1}$. WKK 2924 $\left(\mathrm{S} 8,58^{\prime \prime} \times 22^{\prime \prime}\right)$ is a more likely candidate for the signal at $v=3410 \mathrm{~km} \mathrm{~s}^{-1}$. HIPASS also shows the latter detection at the position of WKK 2924, while nothing can be seen at the position of WKK 2938.

WKK 2993 has a close companion (Woudt \& Kraan-Korteweg 2001) which might contribute to the signal.

WKK 3002/WKK 3006: the two detections in the beam of WKK 3002 can not unambiguously identified: WKK 3002 (SL?, $\left.56^{\prime \prime} \times 20^{\prime \prime}\right)$ is more likely to be the stronger signal at $v=$ $3436 \mathrm{~km} \mathrm{~s}^{-1}$ (cf. HIPASS), while the galaxy at $v=2820 \mathrm{~km} \mathrm{~s}^{-1}$ is more likely WKK $3006\left(13^{\prime \prime} \times 8^{\prime \prime}\right.$, no type $)$.

WKK 3023: with $A_{B}=22^{\mathrm{m}}$ the galaxy is unlikely to be real, and nothing is visible on DENIS or 2MASS images.

WKK 4016/WKK 4022: the profile is due to the blending of two signals. HIPASS shows that the high narrow peak at $v \simeq$ $4680 \mathrm{~km} \mathrm{~s}^{-1}$ is WKK 4016 (SL, 67" $\times 48^{\prime \prime}$ ) at $d=12$ '.1, while the broader profile is probably due to WKK 4022 proper (S5, $\left.91^{\prime \prime} \times 34^{\prime \prime}\right)$. Table 1 gives the parameters for the full profile for WKK 4022 and the measurements of the narrow peak alone for WKK 4016; all parameters are uncertain.

WKK 5240: the detection in the beam of WKK 5267 (not detected) at $d=11$.7 is WKK $5240\left(\mathrm{~S}, 157^{\prime \prime} \times 13^{\prime \prime}\right.$; cf. HIPASS $)$. The profile shape is very noisy and the parameters are uncertain.

WKK 5285: this galaxy has been detected in the beams of three other galaxies: in the OFF observations of WKK 5534 $(d=6.5)$ and of WKK $5556(d=2$ !.7), as well as in the beam of WKK 5297 (not detected) at $d=6$ '1. The detection with the smallest distance to the beam centre is listed in Table 1 and shown in Fig. 1, while the detection in the OFF observation of WKK 5524 is least affected by an RFI at $v=$ $5900 \mathrm{~km} \mathrm{~s}^{-1}$ next to the signal. The other measurements are: $v=5631 \mathrm{~km} \mathrm{~s}^{-1}, \Delta V_{50}=353 \mathrm{~km} \mathrm{~s}^{-1}, \Delta V_{20}=383 \mathrm{~km} \mathrm{~s}^{-1}$, $I=15.65 \mathrm{Jy} \mathrm{km} \mathrm{s}^{-1}, \mathrm{rms}=5.3 \mathrm{mJy}$ (in the beam of WKK 5297); and $v=5635 \mathrm{~km} \mathrm{~s}^{-1}, \Delta V_{50}=357 \mathrm{~km} \mathrm{~s}^{-1}, \Delta V_{20}=396 \mathrm{~km} \mathrm{~s}^{-1}$, $I=15.17 \mathrm{Jy} \mathrm{km} \mathrm{s}^{-1}, \mathrm{rms}=3.3 \mathrm{mJy}$ (in the OFF observation of WKK 5534).

WKK 5366: the identification is uncertain: the optical velocity is $4822 \pm 82 \mathrm{~km} \mathrm{~s}^{-1}$ (WK04), but both HIPASS and JS00 confirm the H I signal to be strongest at the position of WKK 5366. Since the extinction here is $A_{B}=3$. 8 , an obscured galaxy close by cannot be excluded.

WKK 5443OFF: the detection found in the OFF observation of WKK 5443 was subsequently searched for the "best" position. It has also been detected by HIZSS and JS00. No galaxy could be found in the optical or NIR (DENIS, 2MASS).

WKK 5562/WKK 5616/WKK 5642: in the observations of both WKK 5562 and WKK 5642 a narrow single peak appears at $v \simeq 4160 \mathrm{~km} \mathrm{~s}^{-1}$. Using HIPASS we determined that this signal most likely comes from WKK 5616, a late-type galaxy $\left(19^{\prime \prime} \times\right.$ $\left.5^{\prime \prime}\right)$ at $d=5.3$ from WKK 5642 (listed in Table 1) and $d=12.2$ from WKK 5562 with the following parameter: $v=4157 \mathrm{~km} \mathrm{~s}^{-1}$, $\Delta V_{50}=42 \mathrm{~km} \mathrm{~s}^{-1}, \Delta V_{20}=75 \mathrm{~km} \mathrm{~s}^{-1}, I=1.95 \mathrm{Jy} \mathrm{km} \mathrm{s}{ }^{-1}$, $\mathrm{rms}=4.7 \mathrm{mJy}$.

WKK 5595 is included in the catalogue since it is very close to the observed but undetected WKK 5597 (28" $\times 11^{\prime \prime}, \mathrm{L}$ ?) at $d=0.7$ and is of comparable size $\left(30^{\prime \prime} \times 19^{\prime \prime}\right.$, type unknown), that is, the rms can be considered an upper limit for both galaxies.

WKK 5642/WKK 5659: there are three detections in the beam of WKK5642 $\left(48^{\prime \prime} \times 17^{\prime \prime}, \mathrm{SM}\right)$ : the spike at $v=$ $4167 \mathrm{~km} \mathrm{~s}^{-1}$ is WKK 5616 and has been discussed above. The signal at $v=6446 \mathrm{~km} \mathrm{~s}^{-1}$ is assumed to belong to WKK 5642 since it also has two optical velocities of $6045 \pm 42 \mathrm{~km} \mathrm{~s}^{-1}$ (SH92) and $v=6118 \pm 100 \mathrm{~km} \mathrm{~s}^{-1}$ (WK04), though this is only in moderate agreement. WKK $5670\left(24^{\prime \prime} \times 8^{\prime \prime}, \mathrm{SE}:\right)$ at $d=6.6$ with an optical velocity of $v=6329 \pm 44 \mathrm{~km} \mathrm{~s}^{-1}$ 
(WK04) can be excluded as a candidate since it is only $d=5.6$ from WKK 5694, which has also been observed and shows no signal at $v \simeq 6400 \mathrm{~km} \mathrm{~s}^{-1}$. A third signal has been found at $v=4418 \mathrm{~km} \mathrm{~s}^{-1}$ which is likely to be WKK $5659\left(44^{\prime \prime} \times 15^{\prime \prime}\right.$, S6) at $d=3 ! 4$.

IC 4584/IC 4585: this is a detection in the beam of WKK 5581 (not detected). IC 4584 and IC 4585 are two large spiral galaxies at $d \simeq 9^{\prime}$ from the pointing. HIPASS confirms their identity but cannot resolve the pair, though clearly both galaxies contribute to the signal. The measurement of the full profile is given in Table 1.

WKK 5729: the three observations of WKK $5733\left(v_{\text {opt }}=\right.$ $6215 \pm 92 \mathrm{~km} \mathrm{~s}^{-1}$, WK99), WKK $5694\left(v_{\mathrm{opt}}=3412 \pm 36 \mathrm{~km} \mathrm{~s}^{-1}\right.$, FH95), and WKK 5709 show a very similar profile both in line width and peak flux at $v=5729 \mathrm{~km} \mathrm{~s}^{-1}, v=5730 \mathrm{~km} \mathrm{~s}^{-1}$, and $v=5723 \mathrm{~km} \mathrm{~s}^{-1}$, respectively. The left horn appears to come from WKK 5768 (also detected in the beam of WKK 5780), which lies at a distance of $d=13.5, d=17 ! 9$, and $d=11$ '. respectively, from the three pointings; the peak flux of this horn varies according to the distance. Due to the similar peak flux and high velocity end of the rest of the profile we conclude that the detected galaxy must lie at a similar distance from these three pointings. WKK $5729\left(48^{\prime \prime} \times 16^{\prime \prime}, \mathrm{SL}\right)$ lies at $d=66^{\prime} 9, d=9^{\prime} .1$, and $d=5$.9 from WKK 5733, WKK 5694, and WKK 5709, respectively. It is a late-type spiral and therefore not visible with 2MASS and DENIS. The signal is too weak to be detectable with HIPASS.

WKK 5768 is detected in the beam of WKK 5780 (not detected) at $d=9 \cdot$.5. The low-velocity horn is also visible in the observations of WKK 5709 ( $d=11^{\prime}$.7; see plot of WKK 5729), WKK $5733(d=13 ! 5)$ and WKK $5694(d=17 ! 9)$.

WKK 5993/WKK 5999: the detection in the observation of WKK 5993 is the blending of two signals. The low-velocity double-horn comes from WKK 5999 (also observed, see Fig. 1), while the high-velocity part is due to WKK 5993. The parameters for WKK 5993 in Table 1 have been measured by cutting off the profile of WKK 5999 at $v \simeq 3350 \mathrm{~km} \mathrm{~s}^{-1}$. All the parameters are uncertain since the low-velocity end of WKK 5993 is undetermined. For the WKK 5999 profile we have measured $v=3261 \mathrm{~km} \mathrm{~s}^{-1}, \Delta V_{50}=180 \mathrm{~km} \mathrm{~s}^{-1}, \Delta V_{20}=226 \mathrm{~km} \mathrm{~s}^{-1}$, $I=8.83 \mathrm{Jy} \mathrm{km} \mathrm{s}^{-1}, \mathrm{rms}=2.3 \mathrm{mJy}$.

WKK 6187 is included in the catalogue since it is very close to the observed but undetected WKK $6189\left(13^{\prime \prime} \times 8^{\prime \prime}\right.$, E) at $d=$ 0 .9 and is slightly larger $\left(22^{\prime \prime} \times 22^{\prime \prime}\right.$, type unknown $)$, that is, the rms can be considered an upper limit for both galaxies.

WKK 6535/WKK 6570: there are two detections in the beam of WKK 6570 . WKK $6535\left(39^{\prime \prime} \times 9^{\prime \prime}\right.$, S5) lies at $d=6.5$ and is likely to be the detection at $v \simeq 4150 \mathrm{~km} \mathrm{~s}^{-1}$. Since WKK $6570\left(60^{\prime \prime} \times 27^{\prime \prime}, \mathrm{S} 3\right)$ is the larger and brighter of the two we have assumed it to be the closer galaxy at $v=2938 \mathrm{~km} \mathrm{~s}^{-1}$, but the identities remain ambiguous.

WKK 6594: the H I galaxy is probably identical with the IRAS galaxy at $d=2 ! 2$ with $v=642 \pm 35 \mathrm{~km} \mathrm{~s}^{-1}$ (SH92).

WKK 6689/WKK 6732: the two galaxies with similar velocities lie 9'.6 apart. The observation of WKK 6732 shows no significant confusion with the signal of WKK 6689 (though the flux density may be uncertain), while the profile for WKK 6689 is more uncertain.

WKK 7287/WKK 7289: the small signal at $v=5740 \mathrm{~km} \mathrm{~s}^{-1}$ detected in the beam of WKK 7289 is probably WKK 7287 at $d=3 ! 3\left(30^{\prime \prime} \times 20^{\prime \prime}, \mathrm{I}\right)$.

WKK 7460/WKK 7463 is an interacting system with a separation of 1.4: WKK $7460\left(198^{\prime \prime} \times 105^{\prime \prime}, \mathrm{SL}\right)$ is the larger component with an optical velocity of $775 \pm 36 \mathrm{~km} \mathrm{~s}^{-1}$ (SH92), while the profile gives $v=842 \mathrm{~km} \mathrm{~s}^{-1}$. Table 1 gives the full parameters for WKK 7460 only, since the contribution by WKK 7463 $\left(82^{\prime \prime} \times 67^{\prime \prime}, S\right)$ is uncertain. However, considering the types and sizes of the two galaxies as well as the H I velocity as compared to the optical of WKK 7460, we can assume that WKK 7463 contributes to the profile.

WKK 7465/WKK 7198: WKK 7198 has been detected in the OFF observation of WKK 7465 at $d=7 ! 2$, and the profiles overlap. The observation of WKK 7198 (see Fig. 1) shows that the profile extends from $v \simeq 3270 \mathrm{~km} \mathrm{~s}^{-1}$ to $\sim 3540 \mathrm{~km} \mathrm{~s}^{-1}$. The profile of WKK 7465 is therefore truncated and no line widths and flux could be derived. The systemic velocity is likely to be higher than the one given.

WKK 7652/WKK 7689: WKK7652 has been detected in the beam of WKK 7689 (not detected) at $d=11$ '2. Optical velocities for this galaxy are $v=1350 \pm 31 \mathrm{~km} \mathrm{~s}^{-1}$ (RC3) and $v=1478 \pm 38 \mathrm{~km} \mathrm{~s}^{-1}$ (WK04), while other H I measurements find $v=1482 \pm 6 \mathrm{~km} \mathrm{~s}^{-1}$ (RC3). While we find $v=1519 \mathrm{~km} \mathrm{~s}^{-1}$, HK01 has detected WKK 7689 at $v=1559 \pm 3 \mathrm{~km} \mathrm{~s}^{-1}$ in H I with the radio telescope at Effelsberg, which has a smaller beam size ( $9^{\prime}$ as compared to $15^{\prime}$ for Parkes). We can therefore not exclude that part of the signal in our observation comes from WKK 7689.

WKK 7776 has also been detected in the beam of WKK 7794 at $d=10$ !. 7 with $v=2790 \mathrm{~km} \mathrm{~s}^{-1}, \Delta V_{50}=45 \mathrm{~km} \mathrm{~s}^{-1}$, $\Delta V_{20}=55 \mathrm{~km} \mathrm{~s}^{-1}, I=4.11 \mathrm{Jy} \mathrm{km} \mathrm{s}^{-1}, \mathrm{rms}=5.6 \mathrm{mJy}$.

\section{Appendix B: Galaxies in the Vela region}

A number of galaxies outside of the Crux and GA region were also observed within this observing programme, i.e., galaxies in the Vela region $\left(245^{\circ} \gtrsim \ell \gtrsim 275^{\circ}\right)$. They were taken from the ZOA deep optical galaxy catalogue (Salem \& Kraan-Korteweg, in prep.; see Kraan-Korteweg \& Lahav 2000, for preliminary results) that covers the region between Puppis (Saito et al. 1991) and the Hydra/Antlia region (Kraan-Korteweg 2000); see Figs. 2 and 3 in Kraan-Korteweg \& Lahav 2000, for an outline of the surveyed area and the distribution of the uncovered galaxies.

The data of the detected $(N=14)$ and non-detected galaxies $(N=15)$ are presented in Tables B.2 and B.3, which are equivalent to Tables 1 and 4 (see Sects. 3 and 4 for the column descriptions). The profiles of the detected galaxies are shown in Fig. B.1, and Table B.1 gives the independent velocity measurements found in the literature for the detected galaxies (see the description of Table 2 in Sect. 3). The reference for KF95 is Kraan-Korteweg et al. (1995).

Table B.1. Comparison of velocities for Vela galaxies.

\begin{tabular}{|c|c|c|c|c|}
\hline $\begin{array}{r}\text { Ident. } \\
\text { (1) }\end{array}$ & $\begin{array}{rc}V_{\text {hel }} & V_{\text {other }} \\
\mathrm{km} \mathrm{s}^{-1} & \mathrm{~km} \mathrm{~s}^{-1} \\
\text { (2) } & \text { (3) }\end{array}$ & $\begin{array}{l}\text { Origin } \\
\text { (4) }\end{array}$ & $\begin{array}{l}\text { Reference } \\
(5)\end{array}$ & \\
\hline SKK259- 15L & 2730 & $2723 \pm 7$ & H I & HIPASS \\
\hline SKK261- 17L & 2979 & $2974 \pm 10$ & H I & HIPASS \\
\hline SKK262- 89L & 3513 & $3451 \pm 50$ & opt & FW98 \\
\hline SKK262- 36L & 4650 & $4651 \pm 9$ & $\mathrm{HI}$ & HIPASS \\
\hline \multirow[t]{2}{*}{ RKK1949 } & 4030 & $4002 \pm 100$ & opt & KF95 \\
\hline & & $4037 \pm 36$ & opt & SH92 \\
\hline SKK263-164L & 4118 & $4116 \pm \quad 1$ & H I & DN96 \\
\hline \multirow[t]{2}{*}{ SKK263-133L } & 1106 & $969 \pm 54$ & opt & FH95 \\
\hline & & $1106 \pm 6$ & H I & HIPASS \\
\hline \multirow[t]{2}{*}{ SKK263- 12L } & 4540 & $4600 \pm 40$ & opt & $\mathrm{RC} 3$ \\
\hline & & $4545 \pm 6$ & $\mathrm{HI}$ & HIPASS \\
\hline
\end{tabular}


A. C. Schröder et al.: Parkes H I observations of galaxies behind the southern Milky Way. II.

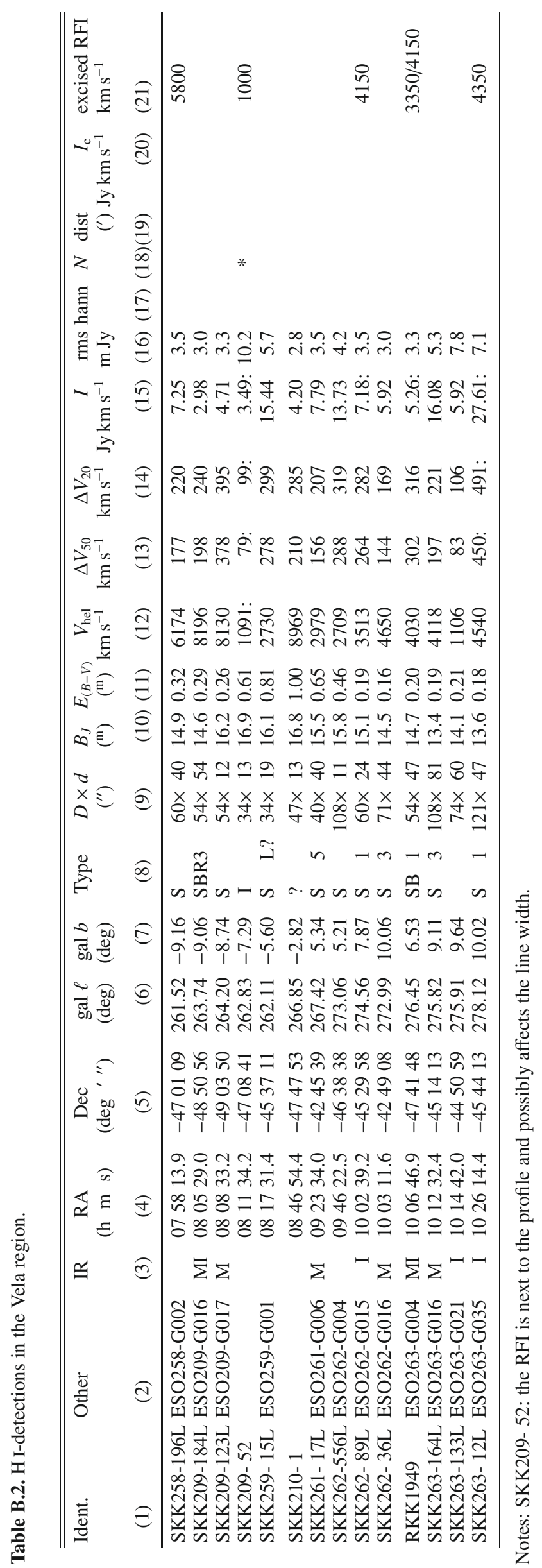

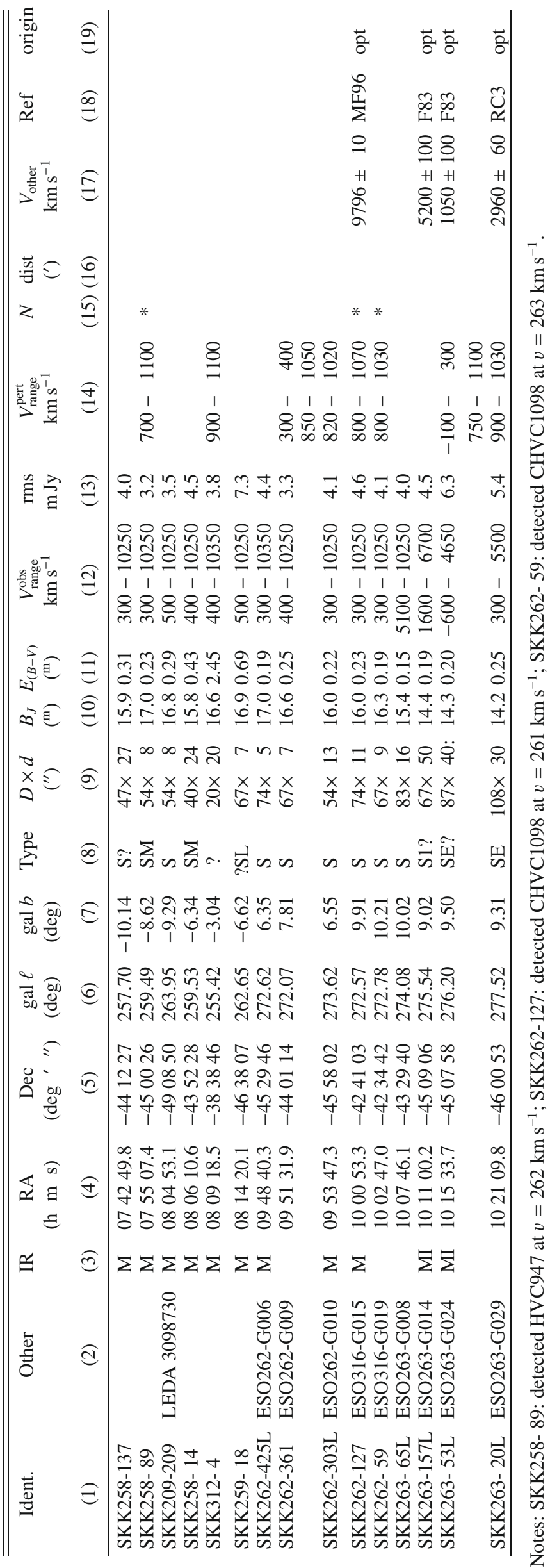



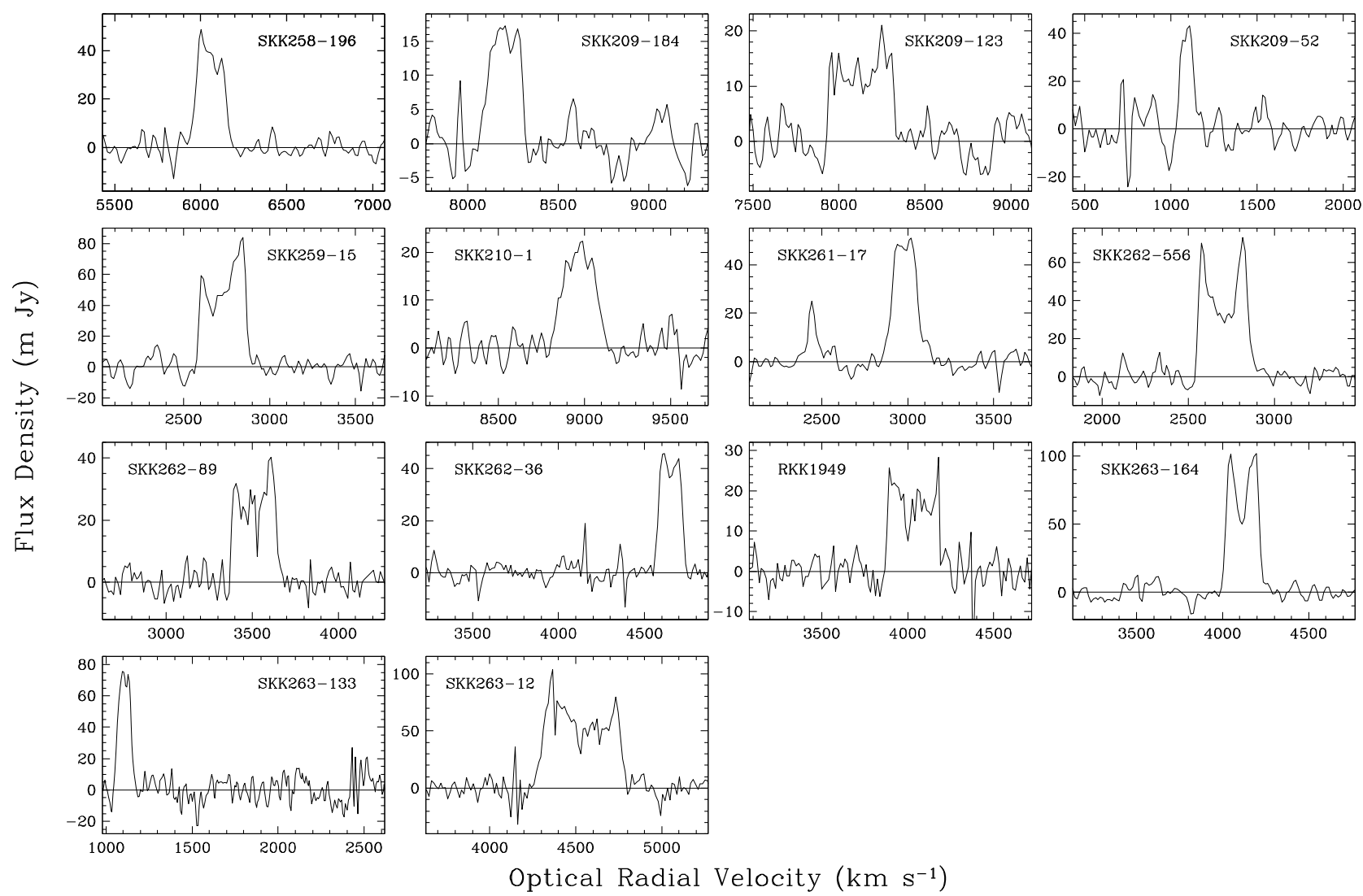

Fig. B.1. H I profiles of the $14 \mathrm{H} \mathrm{I}$ detections in the Vela region. The vertical axis gives the flux density in mJy, the horizontal axis the velocity range (radio convention), generally centred on the radio velocity of the galaxy displaying a width of $1600 \mathrm{~km} \mathrm{~s}^{-1}$. All spectra are baseline-subtracted and generally Hanning-smoothed. The respective identifications are given within the panels. 
A. C. Schröder et al.: Parkes H I observations of galaxies behind the southern Milky Way. II., Online Material p 1
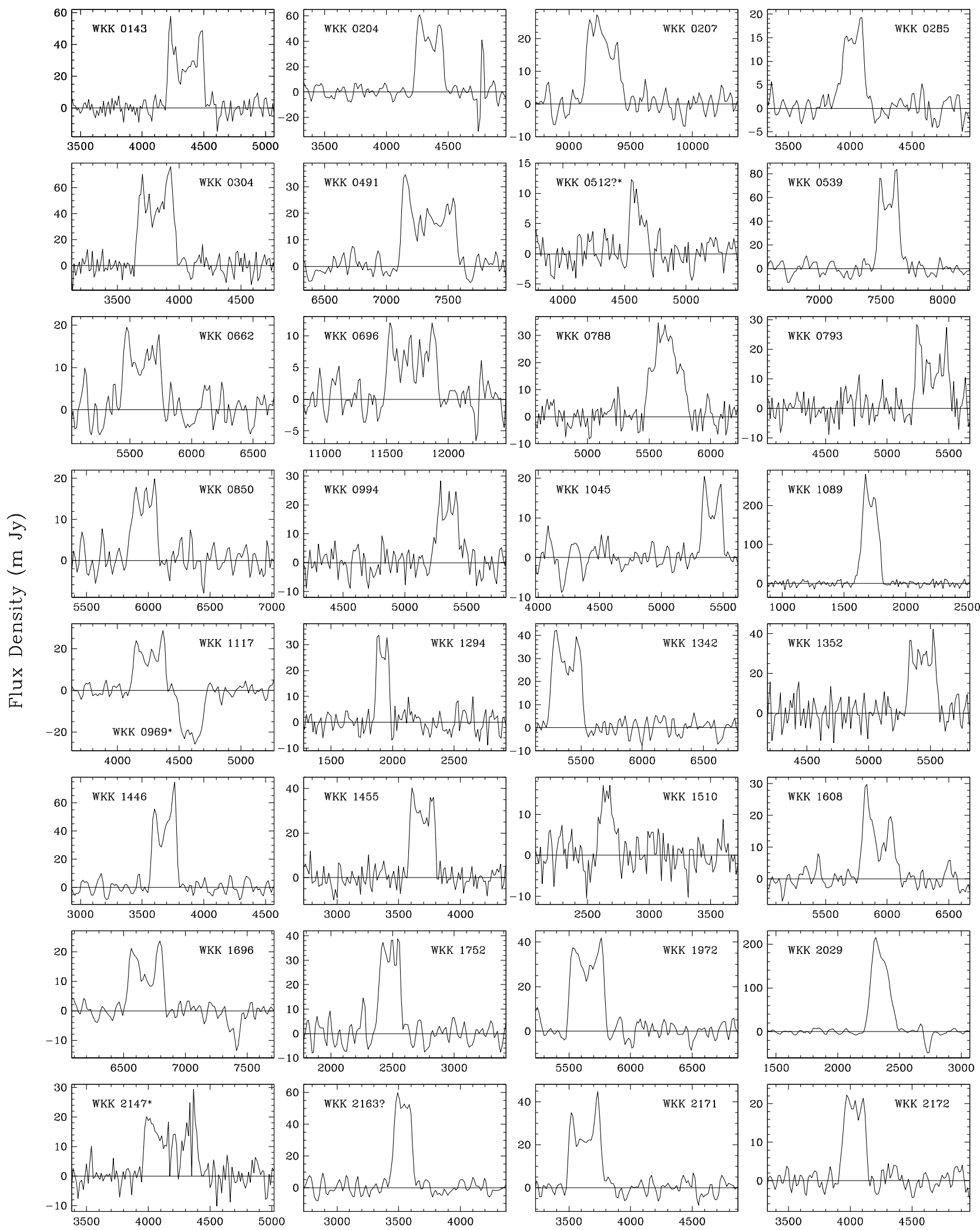

Optical Radial Velocity $\left(\mathrm{km} \mathrm{s}^{-1}\right)$

Fig. 1. Baseline-subtracted H I profiles of the 162 detections in the Crux/GA region. The vertical axis gives the flux density in mJy, the horizontal axis the velocity range (optical convention), generally centred on the velocity of the galaxy and displaying a width of $1600 \mathrm{~km} \mathrm{~s}^{-1}$. All spectra are baseline-subtracted and generally Hanning-smoothed. The identifications are given within the panels. Question marks indicate uncertain identifications, stars denote a detection not at the centre of the beam. 
A. C. Schröder et al.: Parkes H I observations of galaxies behind the southern Milky Way. II., Online Material p 2
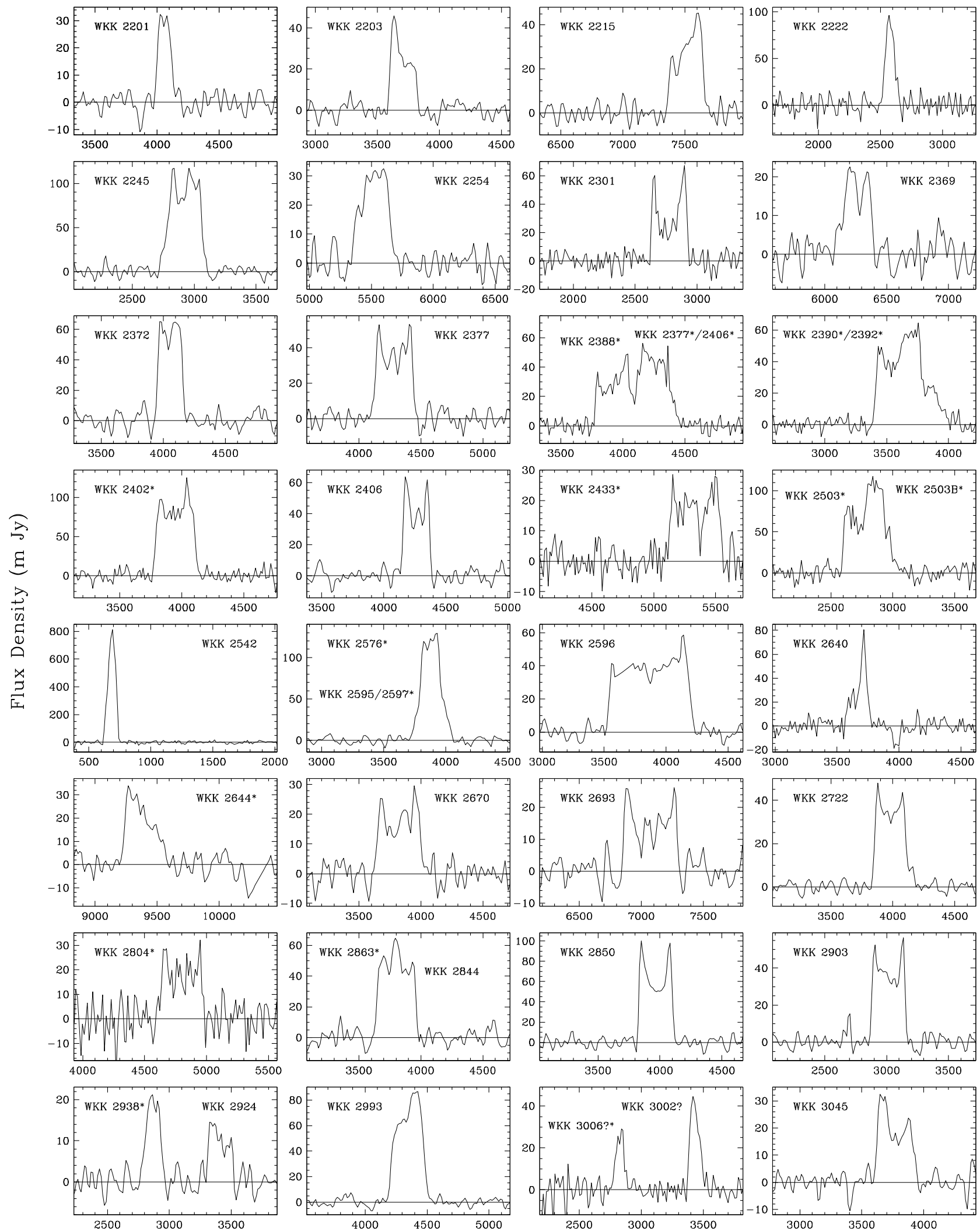

Optical Radial Velocity $\left(\mathrm{km} \mathrm{s}^{-1}\right)$

Fig. 1. continued. 
A. C. Schröder et al.: Parkes H I observations of galaxies behind the southern Milky Way. II., Online Material p 3

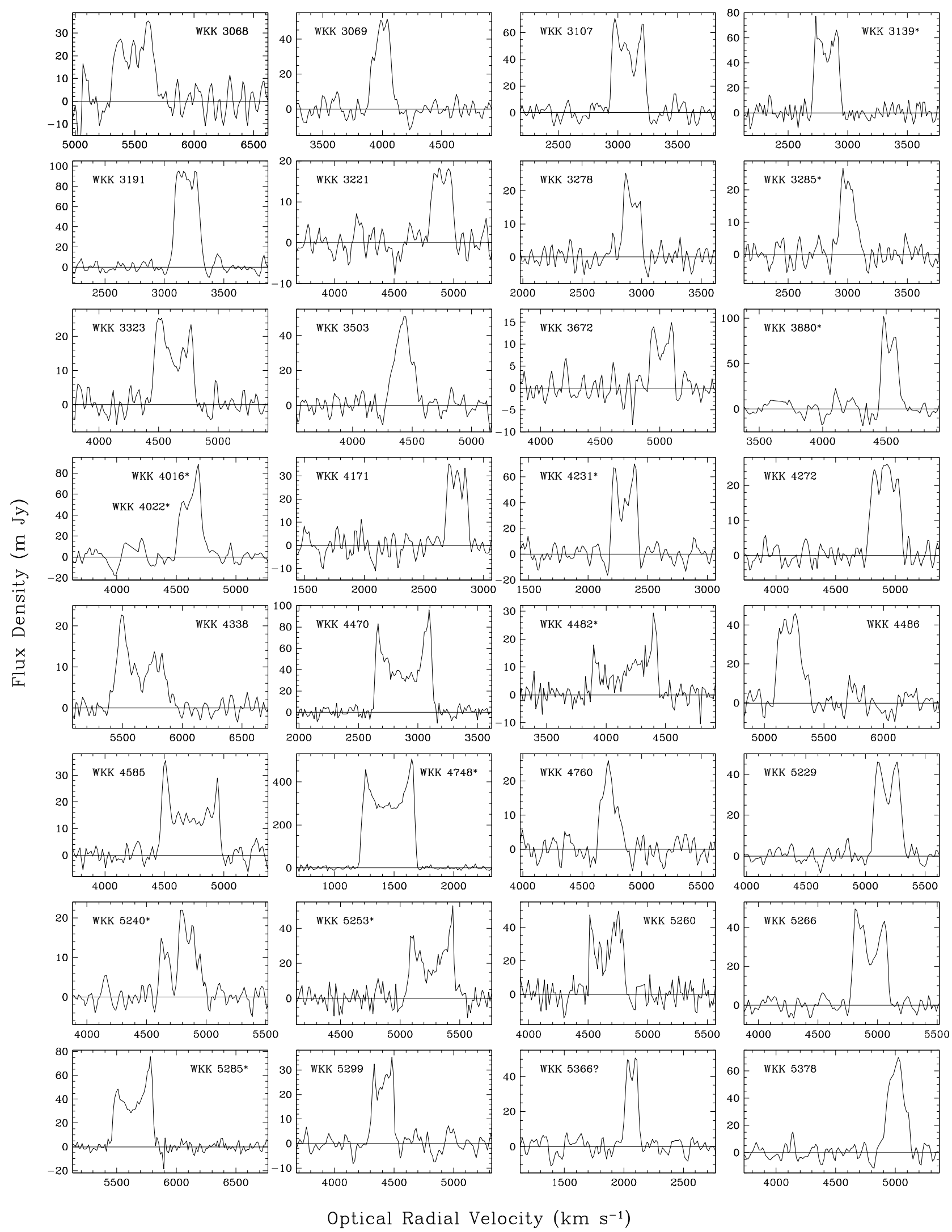

Fig. 1. continued. 
A. C. Schröder et al.: Parkes H I observations of galaxies behind the southern Milky Way. II., Online Material p 4
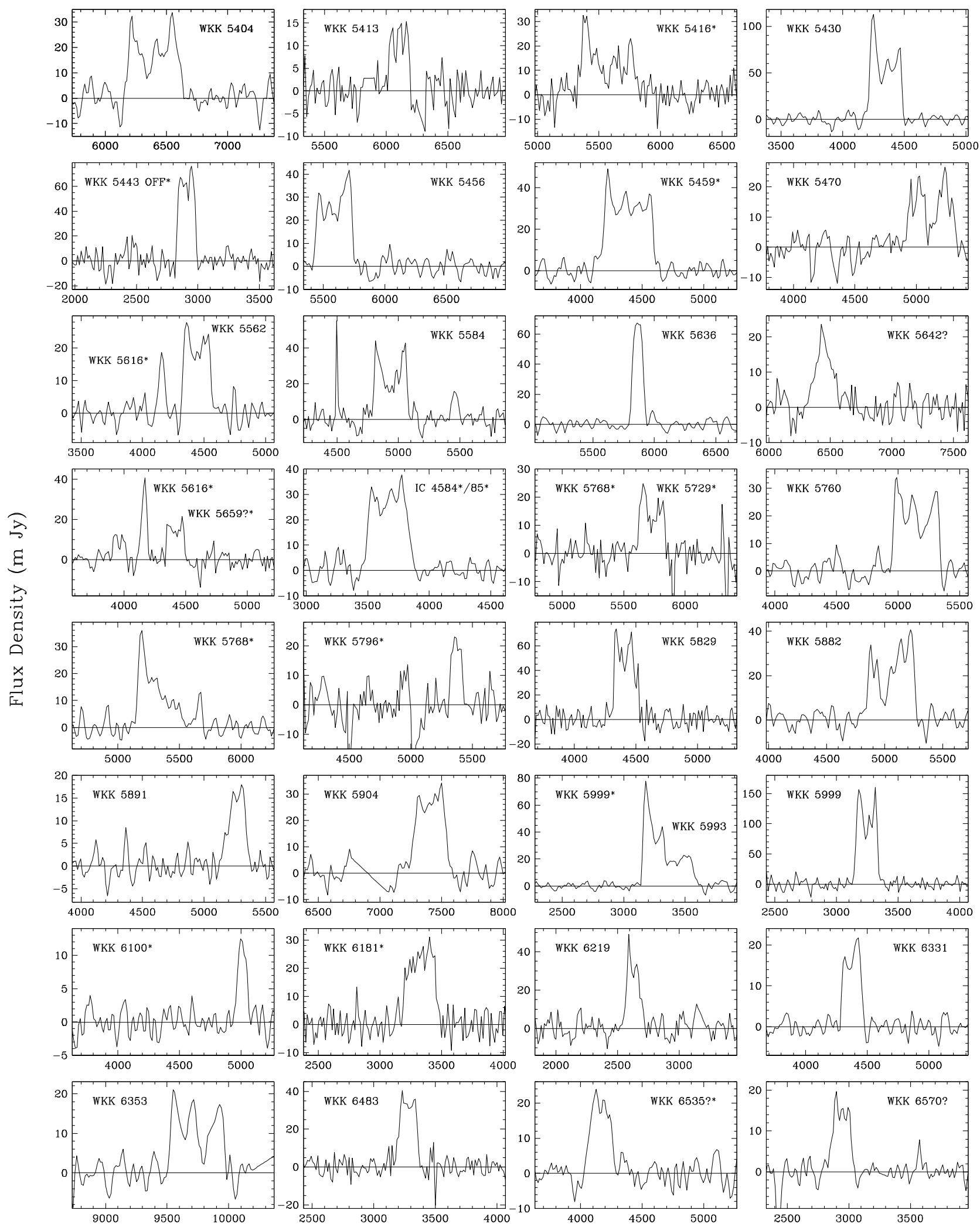

Optical Radial Velocity $\left(\mathrm{km} \mathrm{s}^{-1}\right)$

Fig. 1. continued. 
A. C. Schröder et al.: Parkes H I observations of galaxies behind the southern Milky Way. II., Online Material p 5
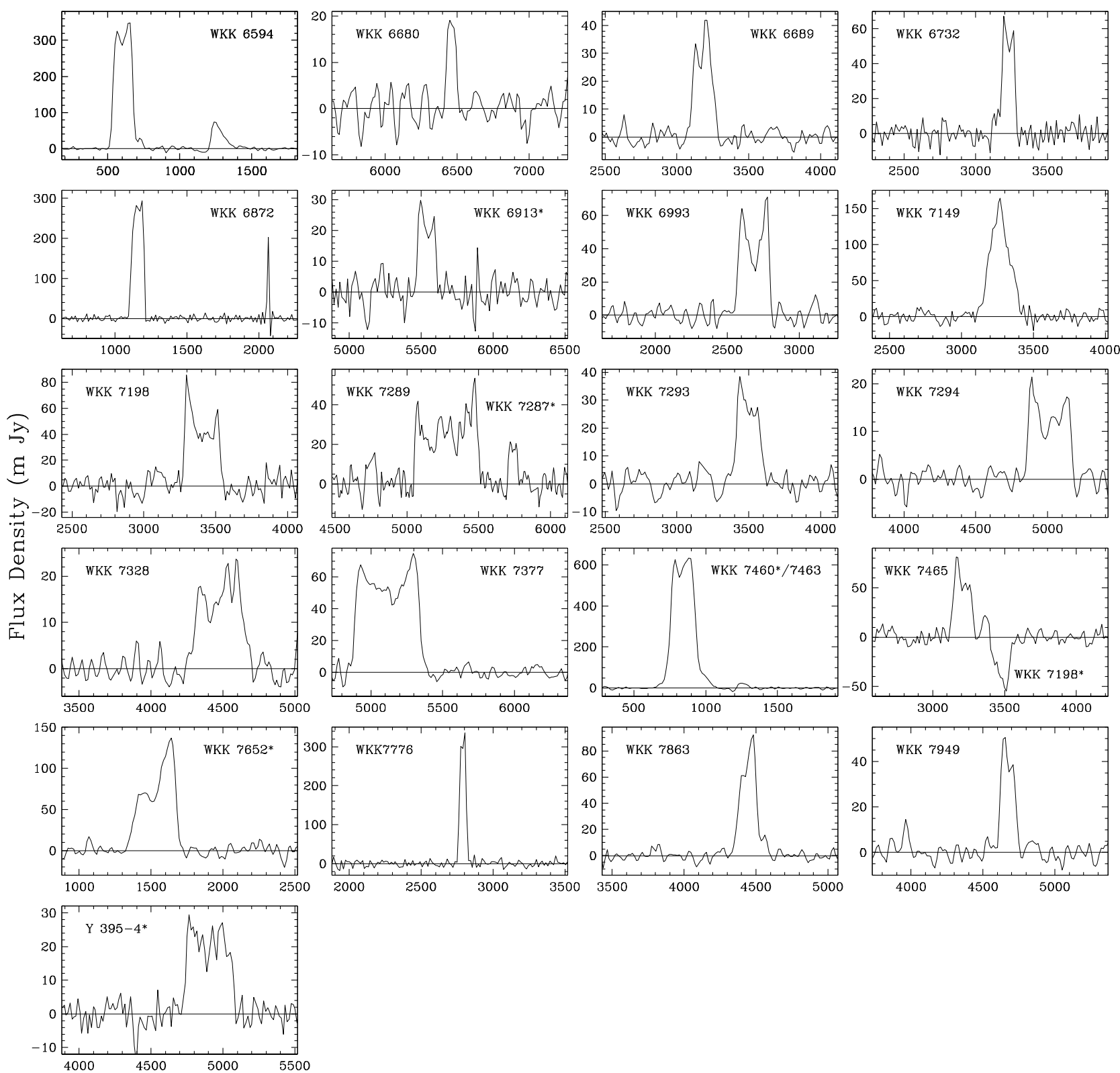

Optical Radial Velocity $\left(\mathrm{km} \mathrm{s}^{-1}\right)$

Fig. 1. continued. 